\title{
The insect somatostatin pathway gates vitellogenesis progression during reproductive maturation and the post-mating response
}

\section{Chen Zhang}

Gwangju Institute of Science and Technology

\section{Crisalejandra Rivera Pérez}

Consejo Nacional de Ciencia y Tecnología, Centro de Investigaciones Biológicas del Noroeste

\section{Fernando Noriega}

Florida International University

Young Joon Kim ( $\nabla$ kimyj@gist.ac.kr)

Gwangju Institute of Science and Technology https://orcid.org/0000-0002-7990-754X

\section{Article}

Keywords: fruit fly, Drosophila melanogaster, sexual maturation, post-mating response.

Posted Date: April 14th, 2021

DOI: https://doi.org/10.21203/rs.3.rs-381466/v1

License: (c) (i) This work is licensed under a Creative Commons Attribution 4.0 International License. Read Full License

Version of Record: A version of this preprint was published at Nature Communications on February 18th, 2022. See the published version at https://doi.org/10.1038/s41467-022-28592-2. 


\section{Abstract}

Oogenesis is closely linked with reproductive maturation and mating status in females. In the fruit fly Drosophila melanogaster, vitellogenesis (yolk accumulation) is an important control point for oogenesis. Vitellogenesis begins upon eclosion and continues through the process of sexual maturation. Upon reaching sexual maturity, vitellogenesis is placed on hold until it is induced again by a mating signal. In flies, this mating signal is sex peptide (SP), a seminal substance that triggers robust egg-laying activity. However, the neural mechanisms that gate vitellogenesis in response to developmental and reproductive signals remain unclear. Here, we have identified a pair of thoracic ganglion neurons that produce the neuropeptide allatostatin $\mathrm{C}$ (AstC-mTh). AstC inhibits the biogenesis of juvenile hormone (JH), a key endocrine stimulator of vitellogenesis. Our genetic evidence indicates that $A s t C$ - $m T h$ neurons gate both the initiation of vitellogenesis that occurs post-eclosion and its re-initiation post-mating. During sexual maturation, which takes place shortly after eclosion, AstC-mTh neurons are activated by excitatory inputs from SP abdominal ganglion (SAG) neurons. In mature virgin females, high sustained activity of SAG neurons seems to shut off vitellogenesis via continuous activation of the AstC-mTh neurons. Upon mating, however, SP inhibits SAG neurons, leading to AstC-mTh neuronal activation. As a result, the inhibition of the CA maintained by the AstC neurons is lifted. This permit both $\mathrm{JH}$ biosynthesis and the progression of vitellogenesis in mated females. Our work has uncovered a central neural circuit that gates the progression of oogenesis during sexual maturation and the post-mating response.

\section{Introduction}

In D. melanogaster, ovary morphogenesis begins in the third larval instar and is completed within 48 hours after pupariation ${ }^{1}$. The ovary contains about 16 ovarioles, each of which represents an independent egg assembly line with progressively developing follicles (egg chambers). This assembly line begins with dividing germline stem cells (GSC) at one end and ends with mature eggs at the other. During oogenesis, a GSC divides asymmetrically to produce a daughter GSC and a cystoblast. The cystoblast subsequently divides four times to produce a cystocyte complex comprising one oocyte and 15 nurse cells. A layer of follicular epithelial cells adheres to and surrounds the oocyte-nurse cell complex to produce a stage 1 follicle. During maturation, follicles undergo sequential transitions from previtellogenesis (stages 1-7) to vitellogenesis (i.e., yolk accumulation) (stages 8-14). In D. melanogaster, females molt into the adult stage (i.e., undergo eclosion) with ovaries that lack vitellogenic follicles ${ }^{2}$. Vitellogenesis begins after eclosion and continues throughout reproductive maturation.

Vitellogenesis initiation is an important control point in oogenesis that integrates hormonal cues to match the female physiological conditions (i.e., nutrition, mating status, etc). The major gonadotropic hormones in $D$. melanogaster are juvenile hormone $(\mathrm{JH})$ and the insect steroid hormones referred to as ecdysteroids ${ }^{3}$. Ecdysteroids stimulate yolk protein (YP) synthesis in the fat body ${ }^{4}$, while $\mathrm{JH}$ stimulates the synthesis and uptake of YP by the ovary ${ }^{4,5}$. Thus, $\mathrm{JH}$ is essential for the continuing development of vitellogenic follicles past stages 8 and 9 . As female fly complete reproductive maturation, JH levels fall 
gradually and vitellogenesis ceases. Later, mating once more stimulates the oogenesis that is required to sustain robust egg-laying activity in mated females. In $D$. melanogaster, a male-derived seminal fluid protein called sex peptide (SP) elicits oogenesis progression ${ }^{6}$. SP induces GSC proliferation by stimulating the biosynthesis of ecdysteroids ${ }^{7}$. The mechanism by which SP regulates $\mathrm{JH}$ biosynthesis and vitellogenesis, however, remains unclear.

The sperm-containing seminal fluid males transfer to females during copulation is composed of a wide range of chemical substances ${ }^{8}$. Not only does seminal fluid provide a supportive milieu for sperm survival, it also modifies the physiology and behavior of recipient females to maximize sperm fertility. SP is the most well-studied seminal fluid substance in D. melanogaster. SP is a 36-mer peptide produced by the male accessory gland ${ }^{9}$ and attached to sperm tails. SP is transferred to females and stored in the female sperm storage organs ${ }^{10}$. Because it is released continuously from sperm by proteolysis of a trypsin-like cleavage site, it can sustain the post-mating state as long as sperm remains in the sperm storage organs (typically about a week). SP acts through SP receptor (SPR)-a G-protein coupled receptor that functions via Gai or Gao-to silence SPR-expressing peripheral sensory neurons (SPSN). SPSNs innervate the lumen of the uterus and send axonal processes into the tip of the abdominal ganglion, relaying the SP signal to Mip-vAL and SAG neurons. Mip-VAL neurons connect SPSNs and SAG neurons in the abdominal ganglion ${ }^{11}$. SAG neurons project to the dorsal protocerebrum in the brain and regulate SP-associated behaviors and physiological responses. These include the suppression of mating receptivity ${ }^{12}$, vaginal plate opening ${ }^{13}$, stimulation of oviposition ${ }^{14}$, increasing salt preference ${ }^{15}$, and reduced siesta sleep ${ }^{16}$. Thus, the SP signal seems to diverge after passing through the SAG neurons to regulate a multitude of post-mating behaviors and physiological changes. The recent discovery of the neural pathways that implicate SAG neurons in oviposition and mating receptivity (i.e., vaginal plate opening) support this notion ${ }^{13,14}$. Still, the circuits downstream of the SAG neurons that regulate vitellogenesis remain unknown.

In this study, we identified two pairs of allatostatin C (AstC)-producing thoracic ganglion neurons (AstC$m T h$ ) that link SAG neurons with JH biosynthesis and vitellogenesis initiation, not only in mated females, but also in young virgin females during reproductive maturation. AstC was first identified in the hawkmoth Manduca sexta based on its "allatostatic" activity (i.e., inhibition of JH biosynthesis) against the $\mathrm{JH}$-producing endocrine organ referred to as the corpora allata $(\mathrm{CA})^{17}$. An allatostatic function was also ascribed to AstC in D. melanogaster when one group found that knockdown of either AstC or AstC receptors increases $\mathrm{JH}$-III levels ${ }^{18}$. In this study, we found that SAG neurons function upstream of AstC$m T h$ neurons, which secrete AstC and inhibit $\mathrm{JH}$ biosynthesis in the CA via AstC receptors (star1 and AstC-R2). As young virgin female complete reproductive maturation, SAG neuron activity rises, augmenting the AstC-induced inhibition of the CA. In mated females, the SP signal attenuates SAG neuron activity and, in turn, the secretion of AstC from AstC-mTh neurons. This reduces AstC-induced inhibition of the $\mathrm{CA}$, eventually permitting $\mathrm{JH}$ biosynthesis and vitellogenic oocyte development.

\section{Results}




\section{AstC gates vitellogenesis during reproductive maturation}

In D. melanogaster, adult females emerge with ovaries lacking vitellogenic follicles (i.e., follicles older than stage 8). To better understand the progression of vitellogenesis that takes place during reproductive maturation, we examined the ovaries of virgin females, counting early (stages 8-11) and late vitellogenic follicles (stages 12-14) separately over 5 days after eclosion. We found control ovaries contained vitellogenic follicles as early as 12 hours post-eclosion (white bar in Fig. 1a). Early vitellogenic follicles accumulated quickly and reached maximum numbers ( $17 \pm 3$ oocytes per female) within 24 hours. They then remained elevated for an additional 24 hours before declining again and reaching a lower basal level thereafter. Later-stage vitellogenic follicles (stages 12-14) began to appear 24 hours after eclosion. They then continued to increase over 3-4 days before reaching a maximum. Thus, vitellogenesis during reproductive maturation seems to progress in two distinct phases. The initial phase, characterized by a rapid accumulation of early vitellogenic follicles, begins soon after eclosion and lasts 48 hours. In the subsequent phase, vitellogenesis initiation slows down until coming to an end 72 hours after eclosion. This maintains the number of early vitellogenic follicles at roughly $31-55 \%$ of its peak.

Considering the role of AstC in generating the circadian vitellogenesis rhythm ${ }^{19}$, we examined posteclosion vitellogenesis in virgin females lacking a functional AstC allele. As with the wild-type controls described above, the ovaries of AstC-deficient $\left(A s t C^{1} / A s t C^{1}\right)$ females contained no vitellogenic follicles when examined immediately after eclosion. Twelve hours later, however, AstC-deficient ovaries produced considerably more early-stage follicles ( $10 \pm 1$ follicles per female) than controls ( $3 \pm 1$ follicles per female). This difference between the two groups reached statistical significance at both 12 and 24 hours after eclosion, but not at 48 hours after eclosion or thereafter (Fig. 1a). Thus, AstC seems to delay the initial phase of vitellogenesis post-eclosion.

Next, we restored AstC expression in AstC-Gal4 cells and again examined vitellogenesis progression posteclosion. As expected, we found restoration of AstC in AstC-Gal4 cells reduced the number of early-stage vitellogenic oocytes to control levels (compare the white and blue bars in Fig. 1a). AstC-Gal4 drives expression in many central nervous system (CNS) neurons ${ }^{19}$. Among these, six pairs of neurons referred to as circadian dorsal neuron 1 (AstC-DN1) are involved in vitellogenesis initiation, specifically with its circadian rhythm. Unlike AstC-Ga/4, however, restoration of AstC expression in AstC-DN1 alone failed to restore the number of early vitellogenic oocytes to control levels (Fig. S1a). Thus, AstC-DN1 is unlikely involved in the vitellogenesis associated with reproductive maturation.

\section{AstC Neurons That Function During Reproductive Maturation}

Consistent with an inhibitory role for the AstC neurons that arrest vitellogenesis associated with reproductive maturation, activation of the AstC-Gal4 neurons of virgin females for three days shortly after eclosion significantly reduces total oogenesis ${ }^{19}$. In an attempt to map the responsible AstC neurons, we restricted the expression of the $A s t C-G a / 4$ driver to distinct subsets of neurons by preparing five additional Gal4 transgenes (AstC-A, AstC-B, AstC-C, AstC-D, and AstC-E), each carrying a 1-kb genomic fragment that together tile the 5 -upstream cis-regulatory region of $A s t C$ used to generate the original $A s t C$-Gal4 
(Fig. 1b). We then used these additional Gal4 drivers to drive expression of the warmth-activated cation channel dTrpA1, thus permitting the specific activation of subsets of the neurons targeted by the original AstC-Gal4 transgene. Among these five new GAL4 lines, AstC-D-Gal4 activation led to a significant reduction in total oogenesis. Females carrying both AstC-D-Gal4 and UAS-dTrpA1 produced $~ 50 \%$ fewer mature eggs at $30^{\circ} \mathrm{C}$ than control females carrying AstC-D-Gal4 alone (Fig. 1C).

Next, we further examined the role AstC-D-Gal4 neurons play in vitellogenesis by driving the expression of the inwardly-rectifying potassium channel Kir2. $1^{20}$ or temperature-sensitive dynamin mutant shibire $\left(S h i^{i s}\right)^{21}$, and counting the number of early and late vitellogenic follicles at 12 and 24 hours after eclosion. Remarkably, we found silencing AstC-D-Gal4 neurons produced a phenocopy of AstC deficiency, leading young virgin females to produce more early-stage vitellogenic follicles (stages 8-11) than controls (Fig. 1d; Fig. S1b). This is precisely what would be expected if these are indeed the AstC neurons of interest-negative regulators of the vitellogenesis associated with reproductive maturation. Thus, we next asked whether the AstC gene is required for the oogenesis inhibition induced by AstC-D-Gal4 neuron activation. Indeed, AstC-D-Ga/4 neuron activation failed to reduce total oogenesis in females lacking a functional AstC allele (Fig. 1e). Moreover, we confirmed that restoring AstC expression only in AstC-D-Gal4 neurons can rescue vitellogenesis in AstC-deficient females to wild-type levels (Fig. 1f). On the strength of these data, we conclude that AstC-D-Gal4 targets a subset of AstC neurons that gates the vitellogenesis associated reproductive maturation.

\section{Identification of AstC-mTh neurons}

To further restrict AstC-D-Gal4 activity to a smaller group of cells, we employed the split-Gal4 system ${ }^{22}$. Genomic DNA fragments used to generate AstC-Gal4 and AstC-D-Gal4 were fused with the Gal4 DNAbinding (DBD) and transcription activation domains (AD), respectively, and combined to produce AstC-Dsplit-Gal4 (i.e., AstC-Gal4DBD; AstC-D-Gal4AD). We found that thermal activation of AstC-D-split-Gal4 neurons suppressed total oogenesis to a level comparable to that of AstC-D-Gal4 neuron activation (i.e., compare Fig. 2a with Fig. 1e). Moreover, when we used AstC-D-split-Gal4 to drive expression of AstC-RNAi, we observed a nearly complete de-repression of the oogenesis inhibition caused by AstC-D-split-Gal4 neuron activation (Fig. 2a). Of note, expression of AstC-RNAi in AstC-D-split-Gal4 neurons also led to a nearly complete loss of anti-AstC expression in the ventral nerve cord (VNC) and dorsal subesophageal zone (SEZ) (red arrows in Fig. S2a).

When we examined the CNS of flies expressing myr-EGFP in AstC-D-split-Gal4 neurons, we found 40 cells with neuron-like morphology (Fig. 2b, c). Of these cells, 6 in the brain and 8 in the ventral nerve cord (VNC) were also positive for anti-AstC (orange circles in Fig. 2c). To visualize and examine the function of $A s t C$ $D$-split-Gal4 neurons in the brain, we combined AstC-D-split-Gal4 with Otd $^{F L P 23,24}$. Unlike the larger population of AstC-D-split-Gal4 neurons, Otd ${ }^{F L P}$-positive brain neurons had a limited impact on total oogenesis (Fig. 2d, Fig. S2b). This suggests the AstC-D-split-Gal4 neurons in the brain are not important for this particular outcome. 
Considering the fact that the silencing of AstC neurons (i.e., AstC-D-Gal4 neurons) seems to expedite posteclosion vitellogenesis by $\sim 12$ hours, AstC neurons associated with reproductive maturation should already be active prior to eclosion. Thus, we monitored neural activity in AstC-D-split-Gal4 neurons using TRIC (i.e., transcriptional reporter of intracellular $\mathrm{Ca}^{2+}$ ), which increases GFP expression in proportion to $\left[\mathrm{Ca}^{2+}\right]_{\mathrm{i}}{ }^{25}$. We observed a robust TRIC signal exclusively in a pair of AstC neurons in the mesothoracic ganglion (hereafter referred to as AstC-mTh neurons) (arrows in Fig. 2e), which remained unchanged in all examined time points from -1 to 3 days post-eclosion (Fig. S2c, Fig. S2d). At low frequency ( 10\%), we observed TRIC labeling of only a single AstC-mTh neuron, revealing its anatomy at single-cell resolution (Fig. 2f). This single AstC-mTh neuron, with its soma located in the mesothoracic ganglion, extensively innervates the dorsal regions of the prothoracic and mesothoracic ganglia and sends an ascending projection that arborizes around the SEZ and inferior dorsal brain. The descending processes from this AstC-mTh neuron do not project beyond the mesothoracic ganglion to the abdominal ganglion.

Next, by driving the expression of the post-synaptic marker DenMark ${ }^{26}$ and the pre-synaptic marker SytGFP $^{27}$ with AstC-D-split-Gal4, we sought to identify, respectively, the inputs and outputs of the AstC-mTh neurons. We observed staining of Syt-GFP indicating the outputs of AstC-mTh in the dorso-lateral mesothoracic ganglion, SEZ, and inferior dorsal regions of the brain (Fig. $2 \mathrm{~g}$ ). DenMark staining was evident in somas, medially running along the mesothoracic ganglion to the SEZ, indicating these as AstC$m T h$ neuron inputs (Fig. 2h). The AstC-mTh neurons and their ascending projection that arborizes the SEZ were evident in both sexes (Fig. 2i, 2j).

\section{AstC-mTh neurons inhibit $\mathrm{JH}$ biosynthesis in the CA}

AstC was initially identified due to its direct allatostatic actions on the CA of the hawkmoth M. sexta ${ }^{17}$. This is why we next asked whether AstC-mTh neurons inhibit $\mathrm{JH}$ production. We measured $\mathrm{JH}$-III levels from female whole-body extracts (Fig. S3). We found that $w^{1118}$ females produced $\sim 10$ times higher $\mathrm{JH}$ III levels on the first day post-eclosion (day 1 ) than during the pupal S8 stage (day - 2). Next, we subjected females expressing dTrpA1 in AstC-D-Gal4 neurons to thermal activation for three days beginning at pupal stage $S 8$ and then measured $\mathrm{JH}$-III levels on day 1 post-eclosion. Indeed, we found thermal activation of AstC-D-Gal4 neurons produced a significant suppression of $\mathrm{JH}$ production. To further understand the role of $A s t C-m T h$ neurons modulating $\mathrm{JH}$ titers, we evaluated $\mathrm{JH}$ signaling by quantifying mRNA levels of Krüppel homolog 1 (Kr-h1), a transcriptional target of $\mathrm{JH}$ signaling ${ }^{28}$. In our previous study, we confirmed that Kr-h1 mRNA levels mirror $\mathrm{JH}$ levels with good sensitivity and fidelity ${ }^{19}$. When we activated AstC-D-Gal4 neurons, we observed a significant reduction in Kr-h1 transcript, to a level approximately $75 \%$ of that of the control group (Fig. 3a). To provide further evidence, we asked how the $\mathrm{JH}$ mimic methoprene affects vitellogenesis in the presence of AstC-D-Ga/4 neuron activation. We found females treated with methoprene showed a significant de-repression of the inhibition of total oogenesis induced by a 3-day thermal activation of AstC-D-Gal4 neurons (Fig. 3b). 
Because activation of AstC-mTh neurons seems to inhibit $\mathrm{JH}$ biosynthesis, we next tested the possibility that the JH-producing CA receives the AstC signal directly. The Drosophila genome contains two AstCreceptor genes, star1 (aka AstC-R1) and AICR2 (aka AstC-R2), both of which encode G-protein coupled receptors highly sensitive to and selective for $A_{s t C}{ }^{29}$. We examined the CA in AstC-R1-Gal4 or AstC-R2Gal4 females, each of which carries an extra exon of the TA-Gal4 transgene in their respective receptor loci ${ }^{19,30}$. Juvenile Hormone Acid O-Methyl Transferase (JHAMT) is the rate-limiting enzyme for JH production, and it is expressed exclusively in the $\mathrm{CA}^{31}$. We found the CA labeled by anti-JHAMT were also positive for both AstC-R1-Gal4 and AstC-R2-Gal4 (Fig. 3c, d). Finally, when we knocked down each receptor in the CA one at a time, we found depletion of each receptor accelerated and increased vitellogenesis in young virgin females, recapitulating the phenotype we observed in those with AstC deficiency or AstC-mTh neuronal silencing (Fig. 3e). When we used JHAMT-Gal4 to drive over-expression of either AstC-R1 or -R2 in the CA, we saw a limited effect on total oogenesis, as measured by the number of mature stage 14 eggs. But simultaneous expression of both AstC-R1 and -R2 in the CA significantly reduced total oogenesis (Fig. 3f). Together, these results suggest the CA is indeed exposed to AstC during reproductive maturation.

\section{AstC-mTh neurons inhibits the CA likely via a hormonal route}

To determine whether AstC-mTh neurons innervate the CA directly, we expressed UAS-myr-EGFP in AstC-Dsplit-Gal4 neurons and could not detect GFP signal around the CA of females (Fig. S4a). Thus, any communication taking place between $A s t C-m T h$ neurons and the $\mathrm{CA}$ is unlikely occurring via a neuronal route. We did, however, notice $A s t C-m T h$ neurons projecting to the dorsomedial SEZ where the aorta contacts the brain (arrow in Fig. $2 f$ and Fig. $2 g$ ). This is reminiscent of the SEZ innervation of insulin producing cells (IPCs), neuroendocrine cells that also stimulate $\mathrm{JH}$ biosynthesis ${ }^{32,33}$. In a previous study, we found that the activation of another subset of AstC neurons (i.e., AstC-DN1p neurons) inhibits JH biosynthesis by suppressing the secretory activity of the IPCs, causing a significant increase in anti-Dilp2 staining as Dilp2 accumulates ${ }^{19}$. For this reason, we tested the possibility that $A s t C-m T h$ neuron activation inhibits IPC activity, affecting JH biosynthesis indirectly. Unlike AstC-DN1 $p$ neurons, however, thermal activation of AstC-mTh neurons had a limited impact on anti-Dilp2 levels in IPCs (Fig. S5a). Inhibition of IPCs or insulin signaling during development typically leads to adults with small body size. And while constitutive activation of AstC-DN1p neurons does indeed produce adults with small body size ${ }^{19}$, similar activation of $A s t C-m T h$ neurons does not measurably affect adult body size (Fig. S5b). Thus, we have concluded that unlike AstC-DN1p neurons, AstC-mTh neurons do not function through the IPCs. Considering the absence of AstC processes innervating the CA, the most parsimonious hypothesis is that $A s t C-m T h$ neurons, like IPCs, also secrete their contents (i.e., AstC) into the circulation to suppress $\mathrm{JH}$ biosynthesis in the CA via a hormonal route.

\section{AstC-mTh neurons play a role in post-mating vitellogenesis}

In D. melanogaster, the mating signal SP stimulates oogenesis progression, specifically vitellogenesis ${ }^{6}$. We reasoned that SP likely does so by inhibiting AstC-mTh neurons, relieving AstC-mediated inhibition of 
the CA. To explore this hypothesis, we first evaluated the activity of AstC-D-split-Gal4 neurons in females before and after mating using TRIC. AstC-mTh neurons in 4-day-old virgin females show strong TRIC signal (Fig. 4a, b), but this is significantly down-regulated within 48 hours after mating with control males, but not after mating with SP-less males (Fig. 4c).

Because SP appears to inhibit the activity of $A s t C-m T h$ neurons, we wanted to determine whether SP stimulates vitellogenesis via the AstC-mTh neurons. We therefore counted vitellogenic follicles (stages 814) and oviposited eggs every four hours after mating for two days (Fig. S6). In control $w^{1118}$ females, we first observed oviposition activity 4 hours after mating, leading to a concomitant reduction in stage 14 eggs. We were unable to observe mating-induced vitellogenesis until 12 hours post-mating, when the number of stage 10 follicles rose compared with virgin controls. Of note, we did not observe any measurable increase in follicles of other stages, whereas stage 10 follicles remained consistently elevated until the end of the experiment. Since it takes $\sim 12$ hours for pre-vitellogenic stage 7 follicles to become stage 10 follicles $^{34}$, the increase in stage 10 follicles observed 12 hours post-mating likely reflects mating-induced vitellogenesis commencing almost immediately after mating. Consistent with this interpretation, females mated with SP-less males showed no increase in stage 10 follicles, as examined 24 hours post-mating (Fig. S6g).

Next, we asked whether AstC-mTh neurons control mating-induced vitellogenesis. Females expressing dTrpA1 in AstC-mTh neurons (i.e., AstC-D-split-Gal4 neurons) were incubated at $30^{\circ} \mathrm{C}$ for 24 hours after mating. We assumed that thermal activation of AstC-mTh neurons would override SP-induced inhibition of AstC-mTh neurons, restoring virgin-like AstC-mTh activity. Indeed, thermal activation blocked matinginduced vitellogenesis, reducing stage 10 follicles by 50\% when compared with controls (Fig. 4d). We also expected $A s t C-m T h$ neuronal silencing would recapitulate mating-induced vitellogenesis even in virgin females. When we blocked the secretory activity of AstC-mTh neurons via expression of the Shits, we were unable to observe any difference in the number of vitellogenic follicles (stages 8-14) in 4-dayold virgin females (Fig. 4e). Thus, we have concluded AstC-mTh neurons do not simulate vitellogenesis per se, but instead gate the vitellogenesis progression that seems to be stimulated by other matingassociated allatotropic factors.

AstC-mTh neurons are interneurons with neurites that innervate the CNS. Because SP inhibits AstC-mTh neurons in the same way it inhibits SAG neurons and other neural components of the SP response circuit ${ }^{11,12}$, we wanted to determine whether $A s t C-m T h$ neurons are functionally linked with SAG neurons. Thus, we prepared female flies expressing TrpA1 in SAG neurons and incubated them at $30^{\circ} \mathrm{C}$ for 24 hours after mating. We expected thermal activation of SAG neurons to override their SP-induced inhibition, thus restoring virgin-like neural activity. Remarkably, we found thermal activation of SAG neurons precisely recapitulated the phenotype induced by thermal activation of $A s t C-m T h$ neurons we described above; it blocked mating-induced vitellogenesis and reduced the number of stage 10 follicles by $~ 50 \%$. Unlike with AstC-mTh neurons, however, activation of SAG neurons also suppressed oviposition, increasing stage 14 eggs in the ovaries. Thus, SAG neurons modulate both vitellogenesis and oviposition, whereas AstC-mTh neurons gate vitellogenesis without affecting oviposition (Fig. 4f). 


\section{The role of SAG neurons in vitellogenesis during reproductive maturation}

Having shown that SAG neurons and AstC-mTh neurons are functional associated in mating-induced vitellogenesis, we wondered whether SAG neurons are also involved in vitellogenesis in young virgin females during reproductive maturation. When we suppressed SAG neuronal activity with Kir2.1 and examined vitellogenesis at 12 and 24 hours after eclosion, we found significantly elevated numbers of early vitellogenic follicles (stages 8-11) just like we observed in females with silenced AstC-mTh neurons (Fig. 5a). Next, we asked whether activation of SAG neurons during reproductive maturation suppresses vitellogenesis and reduces total oogenesis. We found thermal activation of SAG neurons for 3 days posteclosion reduced the number of stage 14 eggs by 50\% (Fig. 5b).

We then wondered whether SAG neurons function through AstC or AstC-mTh neurons. In the absence of a functional AstC allele, thermal activation of SAG neurons failed to suppress total oogenesis (Fig. 5b). Having shown that AstC is required for the function of SAG neurons, we wondered whether AstC-mTh neurons also function downstream of SAG neurons. To test this hypothesis, we activated SAG neurons while also silencing AstC-mTh neurons with Kir2.1. As with AstC-deficiency, the silencing of AstC-mTh neurons also seemed to relieve the vitellogenesis blockade induced by SAG neuron activation, restoring total oogenesis to control levels (Fig. 5c). We then asked whether a JH mimic could override SAG neuron activation and restore total oogenesis to control levels. We found methoprene treatment reversed the vitellogenesis suppression imposed by SAG neuron activation and restored the number of stage 14 eggs to control levels (Fig. 5b). This result provided further evidence of a causal link between SAG neurons and the JH pathway. Finally, we examined SAG neuron activity during reproductive maturation using TRIC analysis (Fig. 5d). As expected, we found evidence of a gradual increase in SAG neural activity the first two days post-eclosion, reaching maximum activity on day 3 when the vitellogenesis associated with reproductive maturation ends (Fig. 1a).

\section{SAG neurons do not form conventional chemical synapses with AstC-mTh neurons}

Having shown that AstC-mTh neurons function downstream of SAG neurons, we wondered how SAG neurons and AstC-mTh neurons interact with one another at the cellular level. By labeling the pre-synaptic terminals of SAG neurons with Syt-GFP, we determined that the SAG outputs arborize near AstC-mTh somas as well as in the mediodorsal SEZ where AstC-mTh neurons project and form extensive postsynaptic terminals (Fig. S7a). Thus, we further explored the possibility that SAG neurons form functional synapses with AstC-mTh neurons by adopting the trans-Tango technique, which visualizes functional post-synaptic neurons ${ }^{35}$. We found SAG neuron expression of trans-Tango labeled many post-synaptic neurons, primarily in the abdominal ganglion. But even after many attempts, we failed to detect transTango activity in AstC-mTh neurons (Fig. S7b). Thus, it seems unlikely that SAG neurons form conventional chemical synapses with AstC-mTH neurons.

\section{Discussion}


Oogenesis is energetically demanding and must be coordinated with ongoing developmental, physiological, and metabolic processes. Vitellogenesis initiation is a critical control point for oogenesis in D. melanogaster. In this species, vitellogenesis begins shortly after eclosion and continues through reproductive maturation, in which females prepare for mating and egg-laying. As females mature over two or three days, vitellogenesis ceases until mating stimulates it again to sustain egg-laying activity. The seminal substance SP acts as a mating signal, stimulating vitellogenesis, ovulation, and oviposition. In this study, we identified a pair of thoracic ganglion neurons (i.e., AstC-mTh neurons) that express the insect somatostatin AstC. We provide evidence that AstC from these neurons modulates vitellogenesis by gating two distinct episodes of JH biosynthesis, one stimulated by adult molting (i.e., eclosion) and the other stimulated by mating. We have also uncovered a functional link between AstC-mTh neurons and SAG neurons, which are a key component of the SP response circuit.

\section{AstC-mTh neurons delay reproductive maturation}

In D. melanogaster, $\mathrm{JH}$ acts on the ovary to initiate vitellogenesis ${ }^{36,37}$. JH levels peak at eclosion (day 0) and decrease gradually over several days ${ }^{38,39}$. $\mathrm{JH}$ titer and therefore oogenesis progression are closely coupled to the endocrine events that induce eclosion in at least two dipteran species, $A$. aegypti and $D$. melanogaster ${ }^{40,41}$. Ecdysis triggering hormone (ETH), which induces molting behaviors (i.e., eclosion) in almost all insect species ever examined ${ }^{42,43}$, also functions as a potent allatotropin. When a pharate adult female is ready to emerge from the pupal case, the endocrine Inka cells secrete ETH. ETH enters the circulation and acts hormonally on the CNS to trigger a sequence of stereotypic motor patterns that culminate in eclosion. When circulating ETH reaches the CA, it triggers the post-eclosion surge of $\mathrm{JH}$. Considering the 28-hour delay required for previtellogenic stage 7 follicles to develop into stage 11 follicles, the ETH-induced $\mathrm{JH}$ surge at day 0 is likely responsible for the marked increase of early vitellogenic follicles (stages 8-11) detected at day 1. In this study, we found that AstC-deficiency in AstC$m T h$ neurons and silencing AstC-mTh neurons both advance vitellogenesis initiation by $\sim 12$ hours. This suggests AstC-deficiency must also advance the JH peak by $\sim 12$ hours, and that AstC-mTh neurons are involved in a temporal decoupling between endocrine events associated with eclosion and reproductive maturation. It is unclear why Drosophila has evolved a mechanism to delay reproductive maturation. In addition to vitellogenesis, $\mathrm{JH}$ also stimulates other processes associated with reproductive maturation, such as pheromone production and the development of mating receptivity ${ }^{44}$. In the wild, D. melanogaster males often wait for females to emerge from their pupal cases before forcefully mating with them ${ }^{45,46}$. We speculate a programmed delay in the processes required for developing attractiveness toward males and mating receptivity would contribute to female fitness by increasing the temporal window in which females can select the best available suitor. The time required for females to reach reproductive maturity varies across Drosophila species. For example, D. pachea females require weeks to become ready to mate, whereas $D$. mettleir females are ready to mate within hours of eclosion. Thus, a comparative analysis of AstC's role in programming the delays required for reproductive maturation across species would be of the great interest ${ }^{47}$. 
As with AstC-deficiency, SAG neuron silencing also advanced post-eclosion vitellogenesis by $\sim 12$ hours. In contrast, activation of SAG neurons during reproductive maturation (i.e., for 3 days post-eclosion) reduced oogenesis by $\sim 50 \%$, precisely phenocopying $A s t C-m T h$ neuron activation. We also found $A s t C-$ $m T h$ neuron silencing blocks the oogenesis-suppressing effect of SAG neuron activation. This epistatic relationship between SAG neurons and AstC-mTh neurons strongly supports the hypothesis that AstC$m T h$ neurons function downstream of SAG neurons. Using the TRIC technique, we found a gradual increase in intracellular $\mathrm{Ca}^{2+}$ in the SAG neurons over the 3 days following eclosion, indicating increased activity. This is consistent with the significant levels of spontaneous firing observed in patch recordings from the SAG neurons of $4-5$-day-old virgin females ${ }^{12}$. We propose that as females undergo reproductive maturation, SAG neurons augment the excitatory inputs into AstC-mTh neurons, driving them to secrete more AstC and cause further inhibition of $\mathrm{JH}$ biosynthesis. Unlike what we observed with the SAG neurons, however, we did not see a progressive increase in TRIC staining of AstC-mTh neurons. But the TRIC staining we observed was so strong that it labeled the entire neuronal arbor of each AstC-mTh neuron. This suggests that a ceiling effect may have masked any further increase in TRIC activity associated with reproductive maturation.

Although we found AstC-deficiency significantly advanced vitellogenesis, it had a limited effect on total oogenesis in virgin females. This is probably because virgin females have limited pre-vitellogenic follicles that can enter vitellogenesis. Consistent with this interpretation, JH has no effect on GSC proliferation ${ }^{7}$. SP stimulates the ovary to produce ecdysteroids, which in turn, stimulate GSC proliferation and presumably pre-vitellogenic follicles. Moreover, there are additional control points and feedback mechanisms that regulate oogenesis progression. For example, ovulation seems to stimulate oocyte maturation. In the absence of ovulation, each ovariole accumulates no more than two or three mature oocytes. Thus, future studies should address whether ovulation modulates the activity of AstC-mTh neurons to stimulate $\mathrm{JH}$ production and vitellogenesis progression.

\section{AstC-mTh neurons in mating-induced vitellogenesis}

An ex vivo analysis found that synthetic SP can induce JHB3 biosynthesis in isolated CA from 3-4-dayold virgin females ${ }^{48}$. Subsequently, SP was implicated in mating-induced vitellogenesis, which was characterized by a pronounced increase of vitellogenic stage 10 follicles $^{6}$. In this study, we confirmed the stimulatory role of SP in mating-induced vitellogenesis by repeating the previous observation in the $w^{1118}$ background, from which most of the fly stocks used in this study were derived (Fig. S6). When SP from the male ejaculate was detected in the hemolymph of mated females, it was proposed to enter the circulation and hormonally stimulate the $\mathrm{CA}^{49}$. More recent studies since the discovery of SPR, however, have suggested SP acts mainly through neuronal pathways comprising SPSN neurons, Mip-vAL neurons, SAG neurons, and the $\mathrm{pC} 1$ neurons ${ }^{11,12,14}$. Of particular note, targeted expression of membrane-tethered $\mathrm{SP}$ (mSP) in uterine SPR neurons (i.e., SPSN neurons) induces virgin females to lay a large number of unfertilized eggs ${ }^{50,51}$. In other words, when $\mathrm{mSP}$ can activate SPR only in the neurons that express it without entering circulation, virgin female egg-laying was found to resemble that of mated females. Thus, 
it is likely that mSP stimulates vitellogenesis exclusively through a neuronal route through the SPSN neurons. Furthermore, electric silencing of either SPSN neurons or SAG neurons (i.e., both mimicking SPR activation) also stimulates robust egg-laying in virgin females at a level comparable to that of mated females $^{12}$. The silencing of $A s t C-m T h$ neurons, however, failed to stimulate vitellogenesis in virgin females. Moreover, the activation of SAG neurons or AstC-mTh neurons led to a significant 50\% reduction in post-mating vitellogenesis. This means it is much more likely that mating-induced vitellogenesis is regulated by allatotropic signals under the control of the neuronal SP response pathway. For example, SP induces 20E biosynthesis via the neuronal SP response pathway ${ }^{7}$. This, in turn, activates ETH expression and secretion from adult Inka cells ${ }^{41}$. It remains unclear, however, whether mating or SP causes ETH secretion. Alternatively, yet not mutually exclusively, SP circulating in post-mating females may also play a role in stimulating $\mathrm{JH}$ biosynthesis, as previously proposed ${ }^{48}$. In other insect species, such as the noctuid moth Spodoptera frugiperda and the mosquito A. aegypti, mating-induced vitellogenesis also seems to be induced at least in part by $\mathrm{JH}$ transferred in the male ejaculate $\mathrm{e}^{52,53}$.

Unlike the SAG neurons, which suppress egg-laying activity, AstC-mTh neurons have limited impact on egg-laying per se. Egg-laying is the outcome of a coordinated array of reproductive processes, such as oogenesis (including vitellogenesis), ovulation, and oviposition. All of these processes are triggered by the neuronal SP pathway. For example, the neural circuit that links SAG neurons and oviposition behavior was recently discovered ${ }^{14}$. Activation of these neurons stimulates oviposition only in mated females, not virgin females. This is likely because this circuit is specialized for oviposition and can only function after oogenesis and ovulation. Our work establishes a novel and distinct branch of the neuronal SP pathway that is specialized exclusively for vitellogenesis.

\section{A source of hormonal AstC that acts directly on the CA}

Since Kramer et al. (1991) ${ }^{17}$ discovered AstC in the hawkmoth M. sexta and reported that it inhibits JH biosynthesis in isolated CAs, several subsequent studies implicated AstC in the regulation of biological processes as diverse as circadian rhythmicity, nociception and immunity in Drosophila ${ }^{19,54,55}$. AstC is expressed in a relatively large number of cells in the brain and gut, consistent with its functional pleiotropism. We found recently that AstC in six pairs of DN1p clock neurons in the brain generate the circadian vitellogenesis rhythm in mated females by rhythmically inhibiting the secretion of the insulinlike peptides that stimulate $\mathrm{JH}$ biosynthesis. In this study, we propose that AstC-mTh neurons are a source of AstC that circulates in the hemolymph to regulate $\mathrm{JH}$ biosynthesis associated with reproductive maturation and the post-mating response. We have presented two major lines of evidence. First, AstC$m T h$ neurons, with their somas in the mesothoracic ganglion, project into the dorsal region of the brain's SEZ. The SEZ is also innervated extensively by other neurosecretory neurons such as the IPCs. This anatomical feature suggests $A s t C-m T h$ neurons are neurosecretory, secreting their contents into the hemolymph. This would allow AstC to travel outside the CNS to the CA, which expresses two highly sensitive and selective GPCR-type AstC receptors (i.e., star1 and AstC-R2). In a second, more important line of evidence, we found knockdown of either of these receptors precisely recapitulated the 12 hour- 
advance of vitellogenesis initiation that occurs in females with AstC-mTh neurons that lack AstC or that are silenced by Kir2.1.

\section{A potential link between $A s t C-m T h$ neurons and post-mating immune responses}

In D. melanogaster, mating stimulates the innate immune system and induces the production of diverse antimicrobial peptides (AMP) including Metchnikowin, Diptericin, and Drosomycin, etc. ${ }^{56}$. As with other aspects of the post-mating response, SP is responsible for post-mating AMP induction. Females that mate with males lacking SP do not produce AMPs upon mating, and females that express SP ectopically and constitutively in the fat body produce AMP regardless of their mating status. Genetic evidence suggests SP induces AMP production via the Toll and Imd pathways. Interestingly, AstC was recently found to have an immunosuppressive function, dampening the Imd pathway ${ }^{54}$. Thus, our finding that SP reduces AstC secretion from AstC-mTh neurons offers an additional and indirect route by which SP boosts the innate immune response and AMP production. Nevertheless, SP likely stimulates the innate immune response via multiple pathways. This is because the SP domain that stimulates AMP production has been mapped to the $\mathrm{N}$-terminal hydroxyproline-rich motifs, which is distinct from the C-terminal domain that activates the neuronal SP pathway via SPSN neurons and SAG neurons ${ }^{57,58}$.

\section{Functional Parallels Between The AstC And SST Systems}

Our work highlights striking conservation in the AstC/SST signaling system between insects and mammals. Not only are the mammalian somatostatin (SST) receptors (sstr1-5) orthologous to the Drosophila AstC receptors, but just as AstC inhibits the insect gonadotropin JH, SST inhibits the mammalian gonadotropins follicle-stimulating hormone (FSH) and leutenizing hormone ( $\mathrm{LH})$ via the hypothalamic neuropeptide gonadotropin releasing hormone $(\mathrm{GnRH})^{59}$. In addition, at least one SSTR also functions as an immunosuppressor that inhibits inflammation ${ }^{60}$ (see above).

In this study, we have shown that the insect SST/AstC pathway modulates JH biosynthesis during reproductive maturation and the post-mating response. JH levels are also linked to other processes associated with female reproduction, such somatic organ remodeling ${ }^{61}$, pheromone production, and mating receptivity ${ }^{44}$. Moreover, AstC-mTh neurons are also present in males, in which $\mathrm{JH}$ regulates processes associated with male reproduction like sex pheromone detection ${ }^{62}$ and male accessory gland development ${ }^{63,64}$. Future studies will be necessary to evaluate the roles of the AstC-mTh neurons in these diverse reproductive processes.

\section{Methods}

Fly stocks. Flies were raised at $25^{\circ} \mathrm{C}$ and $60 \%$ humidity under a $12 \mathrm{~h}: 12 \mathrm{~h}$ light:dark cycle on standard fly media. The stocks used in this study were previously reported or obtained from the Bloomington Drosophila Stock Center (BDSC), the Vienna Drosophila RNAi Center (VDRC), or the Korean Drosophila Resource Center (KDRC). These include otd ${ }^{F L 24}, U A S>\operatorname{stop}>m C D 8 G F P^{65}, U A S>s t o p>d T r p A 7^{66}, U A S-$ 
dTrpA $7^{67}$, UASShits 21, SAG (VT50405)-Gal4 ${ }^{12}$, SAG-GAL4-split1-Gal4(VT50405-p65AD; VT7068GALADBD) ${ }^{12}$, SAG (VT50405)-LexA ${ }^{12}, n S y n b-G a l 4$ carrying UAS-Dicer2 and UAS-mCD8-GFP (gifts from Barry J.Dickson, Janelia Research Campus), y1 w* P\{UAS-myrGFP.QUAS-mtdTomato-3xHA\}su(Hw)attP8; $P\left\{\right.$ trans-Tango\}attP40 (trans-Tango) ${ }^{35}$, AstC-R1-Gal4 ${ }^{30}$, AstC-R2-Gal4 ${ }^{30}$, JHAMT-Gal4 ${ }^{31}$, SP-null males $\mathrm{SP}^{0} / \Delta 130^{68}, \mathrm{CNMa}^{-G a / 4^{19}}$ (KDRC stock number, 2002), AstC ${ }^{19}$ (KDRC \#2166), UAS-Kir2. 1 (III) (a gift from Jan Lab, University of California San Francisco), UAS-Dicer2 (VDRC stock number, 24648), UASmCD8::RFP, LexAop2-mCD8::GFP,nSyb-MI::n/sLexADBDo;UAS-p65AD::CaM (BDSC stock number, 61679), UAS-Syt-EGFP (BDSC \#6926), UAS-DenMark (BDSC \#33061), UAS-Kir2.1 (II) (BDSC \#6596), UAS-NaChBac (BDSC \#9466), AstC-IR1 CG14919 (VDRC \#13772), AstC-R1-IR1 CG7285(VDRC \#13560), and AstC-R2-IR1 CG13702 (BDSC \#25940).

Molecular biology. The AstC Gal4 lines were generated by dividing the 5 -upstream region of the AstC coding sequence into five 1-kb tiling fragments. AstC-A-Gal4 (II), AstC-B-Gal4 (II), AstC-C-Gal4 (II), AstCD-Gal4 (II), AstC-D-Gal4AD (III), AstC-E-Gal4 (II), and AstC-Gal4DBD (II) were prepared in gateway vectors as previously described ${ }^{69}$. Each region was amplified via genomic DNA PCR, cloned into the pENTR vector (Invitrogen), and then recombined into pBPGal4.2::VP16Uw for Gal4, pBPp65AD::ZPUw for Gal4AD or pBPZpGAL4DBD::Uw for Gal4DBD. Each of the final plasmid DNAs was injected into $w^{1118}$ flies with specific landing sites on the second (VIE-72A) or third chromosome (VIE-49B) using the ФC31 system. VIE-72A and VIE-29Ba were gifts from Barry J. Dickson, Janelia Research Campus. The genomic fragments and primer sequences used to generate these lines were as follows: AstC-A-Gal4 (CACCtttccacgaatgctatgcaa, ggcgtcggtaaatgagaaaa), AstC-B-Gal4 (CACCatttaccgacgccaatttca, gaaaagccaacagggtggta), AstC-C-Gal4 (CACCtaccaccctgttggcttttc, aaacacggtcgcttaattcc), AstC-D-Gal4 and AstC-D-Gal4AD (CACCaccgtgtttgccaggataat, tctgcatgcaacaggtaagc), AstC-E-Gal4 (CACCtgttgcatgcagatgatt, tactcaccggtcctgtttcg), and AstC-Gal4DBD (CACCtttccacgaatgctatgcaa, tactcaccggtcctgtttcg). UAS-AstC-R1 and UAS-AstC-R2 were generated by cloning the Notl-AstC-ORF-KpnI fragment amplified from cDNA clone RH36507 (AY070699) into the SST13 vector and then inserting it into a specific site on the third chromosome (VIE-49B) using the ФC31 system. The genomic fragments and primer sequences used to generate these lines were as follows: UAS-AstC-R1 (ttagcggcgcaccatgtttacgtggctgatgat, taatctagattacaatctgtctgctgca) and UAS-AstC-R2 (aatgcggccgccaccatggaaggtggatggtgg, taatctagattataagtccgtgtggagcac).

Bioassays. All assays were repeated on at least two different days. To evaluate vitellogenesis progression after eclosion and total oogenesis, freshly eclosed females were placed individually in vials for the indicated times or for three days and their ovaries were dissected in phosphate buffered saline (PBS). Vitellogenic follicles (stages 8-14) or mature eggs (stage 14) in both ovaries were counted under a stereomicroscope. The ovarioles were separated and the stages of the vitellogenic follicles were determined according to the method used by Jia et al. ${ }^{34}$. To evaluate mating-induced vitellogenesis progression, freshly eclosed females were aged in groups of 10 , mated individually with Canton-Smales or $\mathrm{SP}^{0} / \triangle 130$ males in a $1-\mathrm{cm}$ diameter chamber, and placed individually into vials for the indicated times or for 24 hours after mating. Vitellogenic follicles (stages 8-14) and oviposited eggs were counted. For 
the thermal activation or silencing experiments, virgin or mated females kept individually in vials were transferred into a $30^{\circ} \mathrm{C}$ incubator for 24 hours immediately after copulation.

Methoprene treatment. Methoprene (Sigma-Aldrich, catalog number 40596-69-8) was dissolved in 95\% ethanol $(1 \mu \mathrm{g} / \mu \mathrm{l})$ and added to warm fly food medium (in a liquid state) to produce a final concentration of $1.04 \mu \mathrm{l} / \mathrm{ml}^{19,70}$. Flies were fed this food containing $1.04 \mu \mathrm{l} / \mathrm{ml}$ methoprene or $1 \mu \mathrm{l} / \mathrm{ml} 95 \%$ ethanol (vehicle control) individually for 3 days after eclosion.

Immunohistochemistry. Each fly CNS was dissected in PBS and fixed in 4\% paraformaldehyde for 20-30 min at room temperature. After washing with PBST, the samples were then incubated with rabbit anti-GFP antibody (1:1000; Invitrogen, A11122), rabbit anti-AstC antibody (A gift from Dušan Žitňan, Slovak Academy of Sciences), rat anti-HA antibody (1:100; Roche, 11867423001) ${ }^{71}$, rabbit anti-JHAMT (a gift from Ryusuke Niwa, University of Tsukuba), anti-Dilp2 ${ }^{72}$ (a gift from Yu Kweon from Korea Research Institute of Bioscience and Biotechnology) and mouse anti-nc82 antibody (1:50; Developmental Studies Hybridoma Bank) for 48 hours at $4^{\circ} \mathrm{C}$. Alexa Fluor 488-labeled goat anti-rabbit IgG (1:1000; Invitrogen, A11008), Alexa Fluor 568 goat anti-mouse IgG (1:1000; Invitrogen, A11004), and Alexa Fluor 633 goat anti-rat IgG (1:500; Invitrogen, A21094) were used as secondary antibodies for 24 hours at $4^{\circ} \mathrm{C}$. Confocal images were acquired with a Zeiss LSM700/Axiovert 200M (Zeiss) and processed in Image $\mathrm{J}^{73}$.

To quantify anti-Dilp2 fluorescence, we used a method that was previously described ${ }^{19}$. The maximum intensity Z-projections of 15 consecutive confocal stacks (each $5 \mu \mathrm{m}$-thick) covering the somas of all 14 IPCs were merged in Image J. The relative anti-Dilp2 fluorescence intensity of each brain was calculated by setting the average of control brains (UAS-dTrpA1/+) (for Fig S5a) to 100\%.

TRIC analysis. AstC-D-split-Gal4 (AstC-D-Gal4AD; AstC-Gal4DBD) and SAG-Gal4-split1 (VT50405-p65AD; VT7068-GAL4DBD) were crossed with the TRIC transgenes (UAS-mCD8::RFP, LexAop2-mCD8::GFP,nSybMI::n/sLexADBDo;UAS-p65AD::CaM; see above) to quantify post-mating changes in intracellular $\mathrm{Ca}^{2+}$ in AstC-mTh neurons or SAG neurons. CNS tissues from 4-day-old virgin or mated females (2 days postcopulation) were processed as described above (see the Immunohistochemistry section), but not stained with anti-GFP. To quantify EGFP fluorescence, maximum intensity Z-projections of three confocal stacks (15 consecutive sections each $3.82 \mu \mathrm{m}$-thick) covering the entire soma of each neuron were merged in Image $J$. Then, the relative GFP intensity of each soma was calculated by setting the average of the somas in each group from virgin females to $100 \%$.

RT-qPCR. Total RNA was extracted from whole adult female bodies $(n=15)$ using Trizol (Takara) according to the manufacturer's instructions. RNA $(1 \mu \mathrm{g})$ was reverse transcribed with oligo (dT) primers (Promega) and Accupower RT premix (Bioneer). Quantitative RT-PCR reactions were performed in $10 \mu \mathrm{l}$ reaction volumes using an IQTM5 real-time PCR detection system (Bio-rad) with the SYBR Premix Ex taq (Takara) according to the manufacturer's instructions. The gene specific primers for Rp49 and Kr-h1 were described previously ${ }^{19}$ : Rp49 (forward/reverse, 5'- gacgcttcaagggacagtatctg/5'-aaacgcggttctgcatgag) and Kr-h1 (5'-gcccaaatatgaatccgctctacc/5'- gtcgtcgcccttgttcatgta). 
$\mathrm{JH}$ measurement. For $\mathrm{JH}$-III quantification, 10 pupa or adult female flies from the indicated genotypes and stages were homogenized with a glass musher and transfer to a silanized vial. Flies homogenates were processed by the method described by Bergot et al. ${ }^{74}$ that includes an acetonitrile/pentane extraction and $\mathrm{a}_{18}$ solid-phase extraction cartridge purification. The recovered organic fraction was reduced to a volume of a $100 \mu \mathrm{l}$ and the $\mathrm{JH}$ III epoxide ring was opened by the addition of $150 \mu \mathrm{l}$ of sodium sulfide and incubation at $55^{\circ} \mathrm{C}$ for $30 \mathrm{~min}$. Samples were then extracted with hexane; the recovered organic phase $(\sim 500 \mu \mathrm{l})$ was filtered with a Nalgene filter $(0.2 \mu \mathrm{m}$ nylon membrane, \#176), dried under $\mathrm{N} 2$ and stored at $-20^{\circ} \mathrm{C}$ until used. $\mathrm{JH}$ titers from these whole-body extracts were determined using a high performance liquid chromatography coupled to a fluorescent detector protocol (HPLC-FD) ${ }^{75}$.

Wing size measurement. To measure fly wing size, we modified a method that was previously described ${ }^{19}$. Briefly, the right wings of 3 -day-old female flies were dissected and mounted in $70 \%$ glycerol. Images of each wing were taken using a LEICA EZ4E and the LAS V4.10 program. The distance between wing landmarks \#5 and \#13 was measured with ImageJ and used as an indicator of wing size.

Statistical analysis. GraphPad Prism 5 (GraphPad) was used to analyze the data.

\section{Declarations}

\section{Author contributions}

Conceived and designed the experiment: C.Z. and Y.-J.K.; performed the experiments: C.Z.; analyzed the data: C.Z. and Y.-J.K.; contributed reagents and analysis: C.R.-P. and F.N.; wrote the manuscript: C.Z. and Y.-J.K.

\section{Acknowledgements}

We thank S-I. Kang, H-S. Yoon, J. Mun and J. Song for excellent technical assistance, B. J. Dickson (Janelia Research Campus), D. Žitňan (Slovak Academy of Sciences) for reagents. This work was supported by the GIST Research Institute (GRI) grant funded by the GIST in 2021 and by Basic Science Research Programs (NRF-2015K2A1B8046794, NRF-2018R1A2A1A05079359, NRF-2019R1A4A1029724) through the National Research Foundation of Korea (NRF) funded by Ministry of Science and ICT, Republic of Korea. Stocks were obtained from the Korea Drosophila Resource Center (NRF2017M3A9B8069650), the Bloomington Drosophila Stock Center and the Vienna Drosophila Resource Center.

\section{References}

1. Lin, H. The stem-cell niche theory: Lessons from flies. Nat. Rev. Genet. 3, 931-940 (2002).

2. Spradling, A. C. Developmental genetics of oogenesis. in The Development of Drosophila melanogaster (eds. Bate, M., \& Martinez-Arias ,A.), vol. 1, 1-70 (Cold Spring Harbor Laboratory Press, 1993). 
3. Swevers, L., Raikhel, A. S., Sappington, T. W., Shirk, P. \& latrou, K. Vitellogenesis and post-vitellogenic maturation of the insect ovarian follicle. in Comprehensive Molecular Insect Science (eds. Gilbert, L. I., \& latrou, K.) vol. 1, 87-155 (Elsevier, 2005).

4. Jowett, T. \& Postlethwait, J. H. The regulation of yolk polypeptide synthesis in Drosophila ovaries and fat body by 20-hydroxyecdysone and a juvenile hormone analog. Dev. Biol. 80, 225-234 (1980).

5. Postlethwait, J. H. \& Handler, A. M. The roles of juvenile hormone and 20-hydroxy-ecdysone during vitellogenesis in isolated abdomens of Drosophila melanogaster. J. Insect Physiol. 25, 455-460 (1979).

6. Soller, M., Bownes, M. \& Kubli, E. Mating and sex peptide stimulate the accumulation of yolk in oocytes of Droosophila melanogaster. Eur. J. Biochem. 243, 732-738 (1997).

7. Ameku, T. \& Niwa, R. Mating-induced increase in germline stem cells via the neuroendocrine system in female Drosophila. PLoS Genet. 12, E3849-E3858 (2016).

8. Avila, F. W., Sirot, L. K., Laflamme, B. A., Rubinstein, C. D. \& Wolfner, M. F. Insect seminal fluid proteins: Identification and function. Annu. Rev. Entomol. 56, 21-40 (2011).

9. Chen, P. S. et al. A male accessory gland peptide that regulates reproductive behavior of female D. melanogaster. Cell 54, 291-298 (1988).

10. Peng, J. et al. Gradual release of sperm bound sex-peptide controls female postmating behavior in Drosophila. Curr. Biol. 15, 207-213 (2005).

11. Jang, Y. H., Chae, H. S. \& Kim, Y. J. Female-specific myoinhibitory peptide neurons regulate mating receptivity in Drosophila melanogaster. Nat. Commun. 8, 1-12 (2017).

12. Feng, K., Palfreyman, M. T., Häsemeyer, M., Talsma, A. \& Dickson, B. J. Ascending SAG neurons control sexual receptivity of Drosophila females. Neuron 83, 135-148 (2014).

13. Wang, K. et al. Neural circuit mechanisms of sexual receptivity in Drosophila females. Nature 589, 577-581 (2021).

14. Wang, F. et al. Neural circuitry linking mating and egg laying in Drosophila females. Nature 579, 101105 (2020).

15. Walker, S. J., Corrales-Carvajal, V. M. \& Ribeiro, C. Postmating circuitry modulates salt taste processing to increase reproductive output in Drosophila. Curr. Biol. 25, 2621-2630 (2015).

16. Garbe, D. S. et al. Changes in female Drosophila sleep following mating are mediated by SPSN-SAG neurons. J. Biol. Rhythms 31, 551-567 (2016).

17. Kramer, S. J. et al. Identification of an allatostatin from the tobacco hornworm Manduca sexta. Proc. Natl. Acad. Sci. U. S. A. 88, 9458-9462 (1991).

18. Wang, C., Zhang, J., Tobe, S. S. \& Bendena, W. G. Defining the contribution of select neuropeptides and their receptors in regulating sesquiterpenoid biosynthesis by Drosophila melanogaster ring gland/corpus allatum through RNAi analysis. Gen. Comp. Endocrinol. 176, 347-353 (2012).

19. Zhang, C. et al. The neuropeptide allatostatin $C$ from clock-associated DN1p neurons generates the circadian rhythm for oogenesis. Proc. Natl. Acad. Sci. U. S. A. 118, (2021). 
20. Johns, D. \& Marx, R. Inducible genetic suppression of neuronal excitability. J. Neurosci. 19, 16911697 (1999).

21. Kitamoto, T. Conditional modification of behavior in Drosophila by targeted expression of a temperature-sensitive shibire allele in defined neurons. J. Neurobiol. 47, 81-92 (2001).

22. Luan, H., Peabody, N. C., Vinson, C. R. R. \& White, B. H. Refined spatial manipulation of neuronal function by combinatorial restriction of transgene expression. Neuron 52, 425-436 (2006).

23. Gao, Q. \& Finkelstein, R. Targeting gene expression to the head: The Drosophila orthodenticle gene is a direct target of the Bicoid morphogen. Development 125, 4185-4193 (1998).

24. Asahina, K. et al. Tachykinin-expressing neurons control male-specific aggressive arousal in drosophila. Cell 156, 221-235 (2014).

25. Gao, X. J. et al. A transcriptional reporter of intracellular Ca 2+ in Drosophila. Nat. Neurosci. 18, 917925 (2015).

26. Nicolaï, L. J. J. et al. Genetically encoded dendritic marker sheds light on neuronal connectivity in Drosophila. Proc. Natl. Acad. Sci. U. S. A. 107, 20553-20558 (2010).

27. Zhang, Y. Q., Rodesch, C. K. \& Broadie, K. Living synaptic vesicle marker: Synaptotagmin-GFP. Genesis 34, 142-145 (2002).

28. Fichelson, P., Brigui, A. \& Pichaud, F. Orthodenticle and Kruppel homolog 1 regulate Drosophila photoreceptor maturation. Proc. Natl. Acad. Sci. U. S. A. 109, 7893-7898 (2012).

29. Kreienkamp, H. J. et al. Functional annotation of two orphan G-protein-coupled receptors, drostar1 and -2 , from Drosophila melanogaster and their ligands by reverse pharmacology. J. Biol. Chem. 277, 39937-39943 (2002).

30. Kondo, S. et al. Neurochemical Organization of the Drosophila brain visualized by endogenously tagged neurotransmitter receptors. Cell Rep. 30, 284-297.e5 (2020).

31. Wijesekera, T. P., Saurabh, S. \& Dauwalder, B. Juvenile hormone is required in adult males for drosophila courtship. PLoS One 11, (2016).

32. de Velasco, B. et al. Specification and development of the pars intercerebralis and pars lateralis, neuroendocrine command centers in the Drosophila brain. Dev. Biol. 302, 309-323 (2007).

33. Shiga, S. Anatomy and functions of brain neurosecretory cells in diptera. Microsc. Res. Tech. 62, 114-131 (2003).

34. Jia, D., Xu, Q., Xie, Q., Mio, W. \& Deng, W. M. Automatic stage identification of Drosophila egg chamber based on DAPI images. Sci. Rep. 6, 1-12 (2016).

35. Talay, M. et al. Transsynaptic mapping of second-order taste neurons in flies by trans-Tango. Neuron 96, 783-795.e4 (2017).

36. Postlethwait, J. H. \& Shirk, P. D. Genetic and endocrine regulation of vitellogenesis in Drosophila. Integr. Comp. Biol. 21, 687-700 (1981).

37. Bownes, M. The roles of juvenile hormone, ecdysone and the ovary in the control of Drosophila vitellogenesis. J. Insect Physiol. 35, 409-413 (1989). 
38. Bownes, M. \& Rembold, H. The titre of juvenile hormone during the pupal and adult stages of the life cycle of Drosophila melanogaster. Eur. J. Biochem. 164, 709-712 (1987).

39. Sugime, Y. et al. Upregulation of juvenile hormone titers in female Drosophila melanogaster through mating experiences and host food occupied by eggs and larvae. Zoolog. Sci. 34, 52-57 (2017).

40. Areiza, M., Nouzova, M., Rivera-Perez, C. \& Noriega, F. G. Ecdysis triggering hormone ensures proper timing of juvenile hormone biosynthesis in pharate adult mosquitoes. Insect Biochem. Mol. Biol. 54, 98-105 (2014).

41. Meiselman, M. et al. Endocrine network essential for reproductive success in Drosophila melanogaster. Proc. Natl. Acad. Sci. U. S. A. 114, E3849-E3858 (2017).

42. Zitnan, D. \& Adams, M. E. Neuroendocrine regulation of ecdysis. in Insect Endocrinology (ed. Gilbert, L. I. ) 253-309 (Elsevier, 2012).

43. Park, Y., Filippov, V., Gill, S. S. \& Adams, M. E. Deletion of the ecdysis-triggering hormone gene leads to lethal ecdysis deficiency. Development vol. 129 (2002).

44. Bilen, J., Atallah, J., Azanchi, R., Levine, J. D. \& Riddiford, L. M. Regulation of onset of female mating and sex pheromone production by juvenile hormone in Drosophila melanogaster. Proc. Natl. Acad. Sci. U. S. A. 110, 18321-18326 (2013).

45. Markow, T. A. Forced matings in natural populations of Drosophila. Am. Nat. 156, 100-103 (2000).

46. Seeley, C. \& Dukas, R. Teneral matings in fruit flies: Male coercion and female response. Anim. Behav. 81, 595-601 (2011).

47. Markow, T. A. The secret lives of Drosophila flies. Elife 4, 1-9 (2015).

48. Moshitzky, P. et al. Sex-Peptide Activates juvenile hormone biosynthesis in the Drosophila melanogaster corpus allatum. Arch. Insect Biochem. Physiol. 32, 363-374 (1996).

49. Pilpel, N., Nezer, I., Applebaum, S. W. \& Heifetz, Y. Mating-increases trypsin in female Drosophila hemolymph. Insect Biochem. Mol. Biol. 38, 320-330 (2008).

50. Yang, C. hui et al. Control of the postmating behavioral switch in Drosophila females by internal sensory neurons. Neuron 61, 519-526 (2009).

51. Häsemeyer, M., Yapici, N., Heberlein, U. \& Dickson, B. J. Sensory neurons in the Drosophila genital tract regulate female reproductive behavior. Neuron 61, 511-518 (2009).

52. Hassanien, I. T. E., Grötzner, M., Meyering-Vos, M. \& Hoffmann, K. H. Neuropeptides affecting the transfer of juvenile hormones from males to females during mating in Spodoptera frugiperda. $J$. Insect Physiol. 66, 45-52 (2014).

53. Clifton, M. E., Correa, S., Rivera-Perez, C., Nouzova, M. \& Noriega, F. G. Male Aedes aegypti mosquitoes use $\mathrm{JH}$ III transferred during copulation to influence previtellogenic ovary physiology and affect the reproductive output of female mosquitoes. J. Insect Physiol. 64, 40-47 (2014).

54. Bachtel, N. D., Hovsepian, G. A., Nixon, D. F. \& Eleftherianos, I. Allatostatin C modulates nociception and immunity in Drosophila. Sci. Rep. 8,1-11 (2018). 
55. Díaz, M. M., Schlichting, M., Abruzzi, K. C., Long, X. \& Rosbash, M. Allatostatin-C/AstC-R2 Is a aovel pathway to modulate the circadian activity pattern in Drosophila. Curr. Biol. 29, 13-22.e3 (2019).

56. Peng, J., Zipperlen, P. \& Kubli, E. Drosophila sex-peptide stimulates female innate immune system after mating via the Toll and Imd pathways. Curr. Biol. 15, 1690-4 (2005).

57. Domanitskaya, E. V, Liu, H., Chen, S. \& Kubli, E. The hydroxyproline motif of male sex peptide elicits the innate immune response in Drosophila females. FEBS J. 274, 5659-68 (2007).

58. Yapici, N., Kim, Y.-J., Ribeiro, C. \& Dickson, B. J. A receptor that mediates the post-mating switch in Drosophila reproductive behaviour. Nature 451, 33-37 (2008).

59. Bhattarai, J. P. et al. Somatostatin inhibition of gonadotropin-releasing hormone neurons in female and male mice. Endocrinology 151, 3258-3266 (2010).

60. Pintér, E., Helyes, Z. \& Szolcsányi, J. Inhibitory effect of somatostatin on inflammation and nociception. Pharmacology and Therapeutics vol. 112 440-456 (2006).

61. Reiff, T. et al. Endocrine remodelling of the adult intestine sustains reproduction in drosophila. Elife 4, $1-19$ (2015).

62. Anton, S. \& Gadenne, C. Effect of juvenile hormone on the central nervous processing of sex pheromone in an insect. Proc. Natl. Acad. Sci. U. S. A. 96, 5764-5767 (1999).

63. Shemshedini, L., Lanoue, M. \& Wilson, T. G. Evidence for a juvenile hormone receptor involved in protein synthesis in Drosophila melanogaster. J. Biol. Chem. 265, 1913-1918 (1990).

64. Wilson, T. G., DeMoor, S. \& Lei, J. Juvenile hormone involvement in Drosophila melanogaster male reproduction as suggested by the Methoprene-tolerant 27 mutant phenotype. Insect Biochem. Mol. Biol. 33, 1167-1175 (2003).

65. Yu, J. Y., Kanai, M. I., Demir, E., Jefferis, G. S. X. E. \& Dickson, B. J. Cellular organization of the neural circuit that drives Drosophila courtship behavior. Curr. Biol. 20, 1602-14 (2010).

66. von Philipsborn, A. C. et al. Neuronal control of Drosophila courtship song. Neuron 69, 509-522 (2011).

67. Hamada, F. N. et al. An internal thermal sensor controlling temperature preference in Drosophila. Nature 454, 217-20 (2008).

68. Liu, H. \& Kubli, E. Sex-peptide is the molecular basis of the sperm effect in Drosophila melanogaster. Proc. Natl. Acad. Sci. U. S. A. 100, 9929-33 (2003).

69. Pfeiffer, B. D. et al. Refinement of tools for targeted gene expression in Drosophila. Genetics 186, 735-55 (2010).

70. Flatt, T. \& Kawecki, T. J. Juvenile hormone as a regulator of the trade-off between reproduction and life span in Drosophila melanogaster. Evolution (N. Y). 61, 1980-1991 (2007).

71. Hampel, S. et al. Drosophila Brainbow: A recombinase-based fluorescence labeling technique to subdivide neural expression patterns. Nat. Methods 8, 253-259 (2011).

72. Kwak, S. J. et al. Drosophila adiponectin receptor in insulin producing cells regulates glucose and lipid metabolism by controlling insulin secretion. PLoS One 8, 2-11 (2013). 
73. Schneider, C. A., Rasband, W. S. \& Eliceiri, K. W. NIH Image to ImageJ: 25 years of image analysis. Nat. Methods 9, 671-675 (2012).

74. Bergot, B. J. Method for quantitative determination of the four known juvenile hormones in insect tissue using gas chromatography-mass spectroscopy. J. Chromatogr. 23-244 (1981).

75. Rivera-Perez, C., Nouzova, M. \& Noriega, F. G. A quantitative assay for the juvenile hormones and their precursors using fluorescent tags. PLoS One 7, e43784 (2012).

\section{Figures}




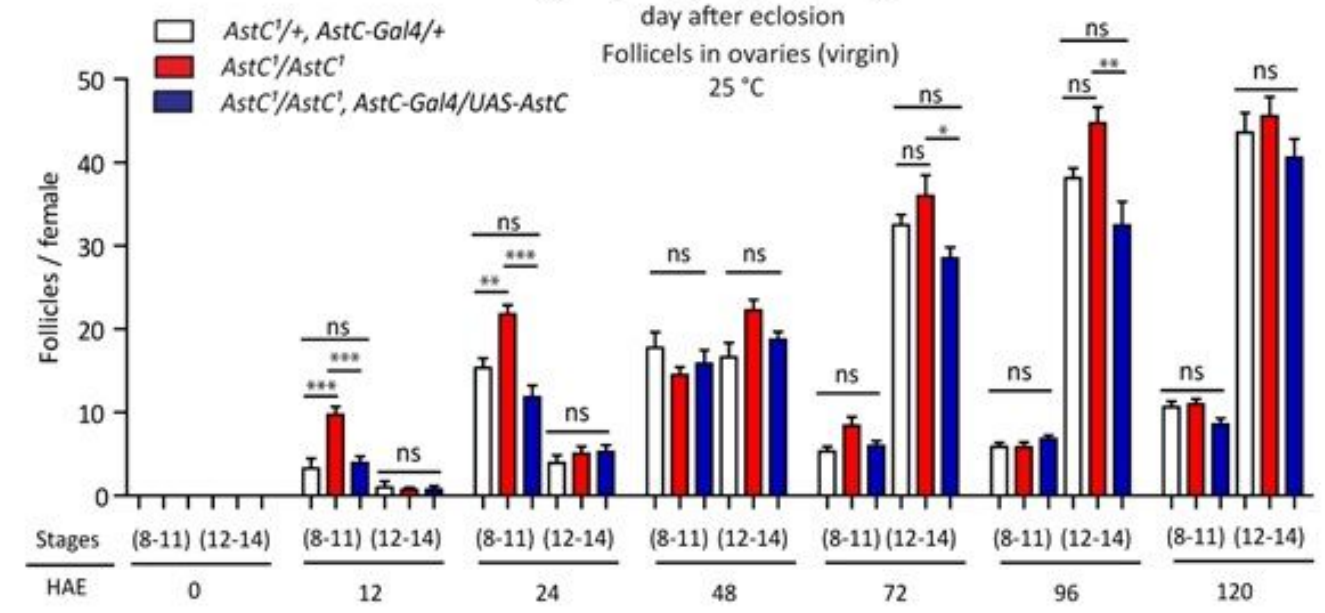

b

C

Examining ovaries
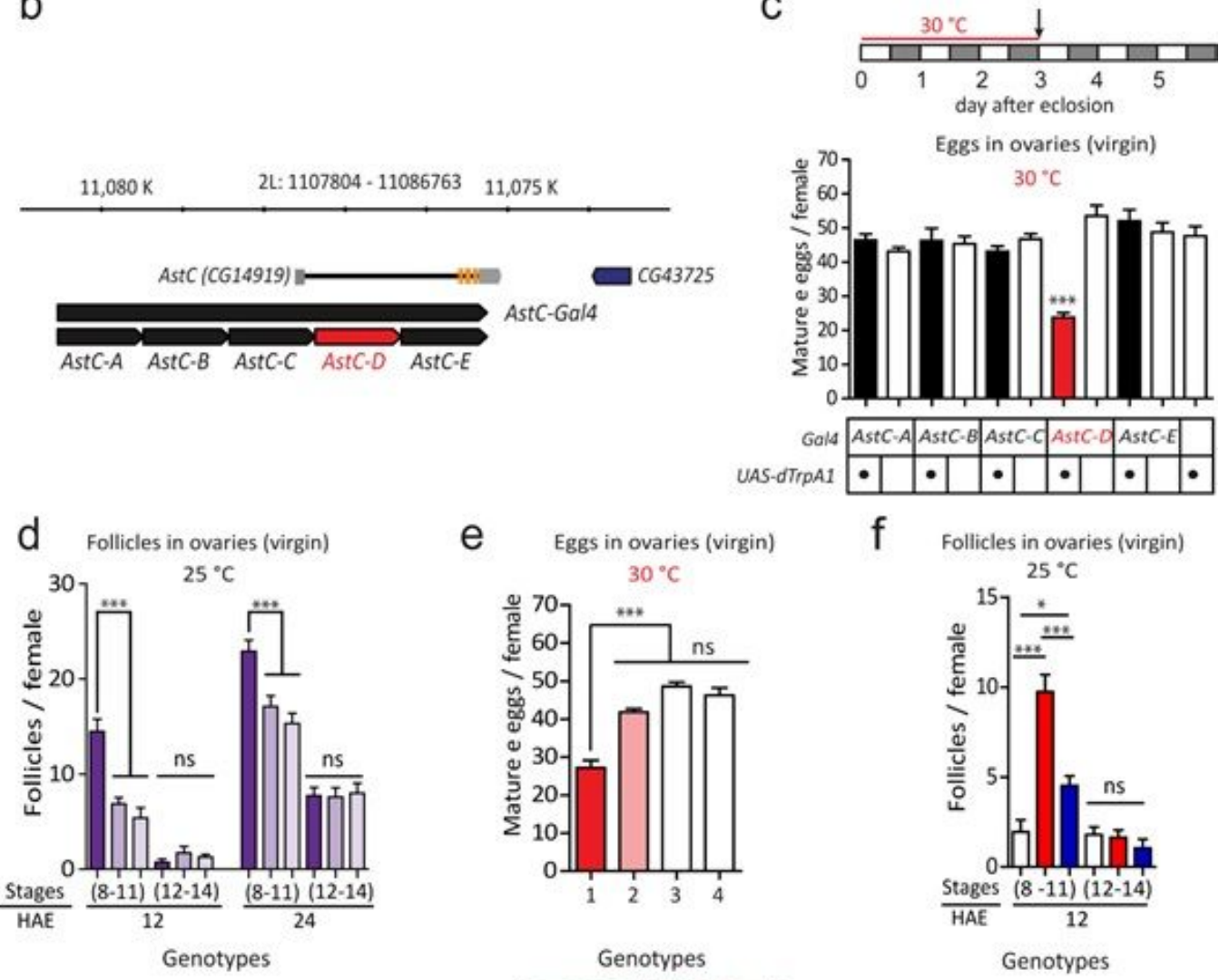

e

f Follicles in ovaries (virgin)
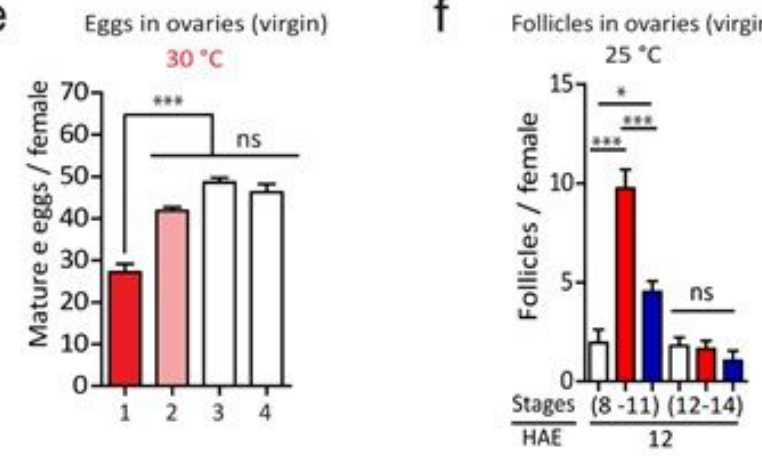

Genotypes

1: AstC-D-Gal4/UAS-dTrpA1, AstC $1 /+$

2: AstC-D-Gal4/UAS-dTrpA1, AstC' $/$ AstC

3: $A s t C^{\prime} /+\quad 4: A s t C^{\prime} / A s t C^{\prime}$

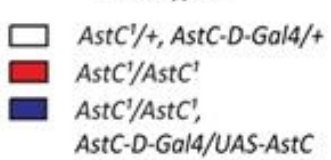

\section{Figure 1}

AstC from AstC-D-Gal4 neurons gates vitellogenesis during reproductive maturation. a Above, experimental protocol for $a, d$, and $f$. Below, number of stage 8-11 and 12-14 follicles per virgin female of the indicated genotypes at the indicated hours after eclosion (HAE) $(n=8-12)$. One-way ANOVA followed by Tukey's test for multiple comparisons ( $a, d, e$, and f); ${ }^{\star \star \star} p<0.001$; ${ }^{\star \star} p<0.005$; ns (non-significance) $p>$ 0.05. b AstC gene structure and genomic fragments (black or red bars) used to generate AstC-Gal4 and 
the other related Gal4, Gal4AD, and Gal4DBD transgenes. c Above, experimental protocol for $c$ and e. Below, number of mature eggs per virgin female 3 days after eclosion of the indicated genotypes at $30^{\circ} \mathrm{C}$ $(n=40-60)$. Unpaired t-test; ${ }^{\star \star *} p<0.001$ compared with Gal4 control (white bar); no labeling, $p>0.05 . d$ Number of stage 8-11 and 12-14 follicles per virgin female of the indicated genotypes at the indicated HAE $(n=8-12)$. e Number of mature eggs per virgin female of the indicated genotypes 3 days after eclosion at $30^{\circ} \mathrm{C}(n=40-60)$. $f$ Number of stage $8-11$ and $12-14$ follicles per virgin female of the indicated genotypes at $12 \operatorname{HAE}(n=8-12)$.
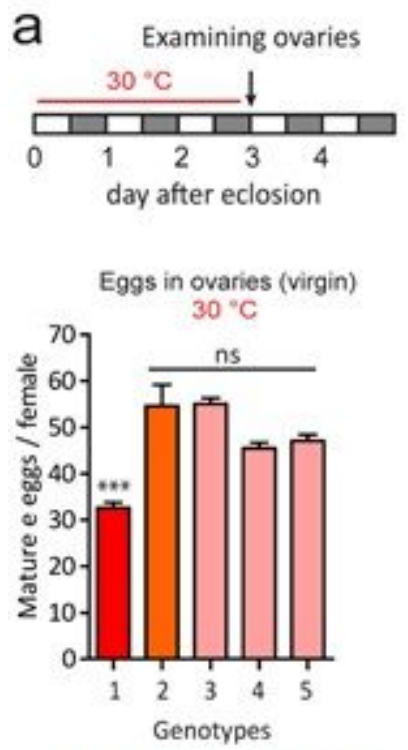

1: AstC-D-split-Gol4/UAS-dTrpA1 2: AstC-D-split-Gol4/UAS-dTrpA1 ,UAS-AstC-AR

3: AstC-D-split-Gal4/*

4: $+/$ UAS-dTrpA1, +/UAS-AstC-IR

$5:+/$ UAS-dTrpA1

d Eggs in ovaries (virgin) $30^{\circ} \mathrm{C}$

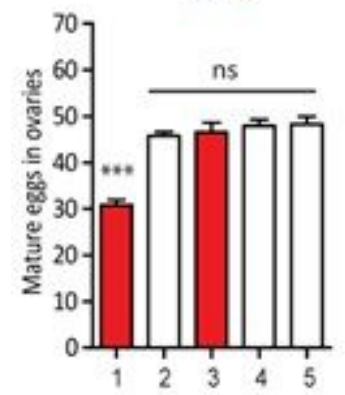

Genotypes

1: AstC-D-split-Gol4/UAS-dTrpA1

2: +/UAS-dTrpA1

3: AstC-D-split-Gal4/

UAS<stop<dTrpA1, Otd-Flp

4: +/UAS<stop<dTrpA1, Otd-Flp

5: AstC-D-split-Gal4/+
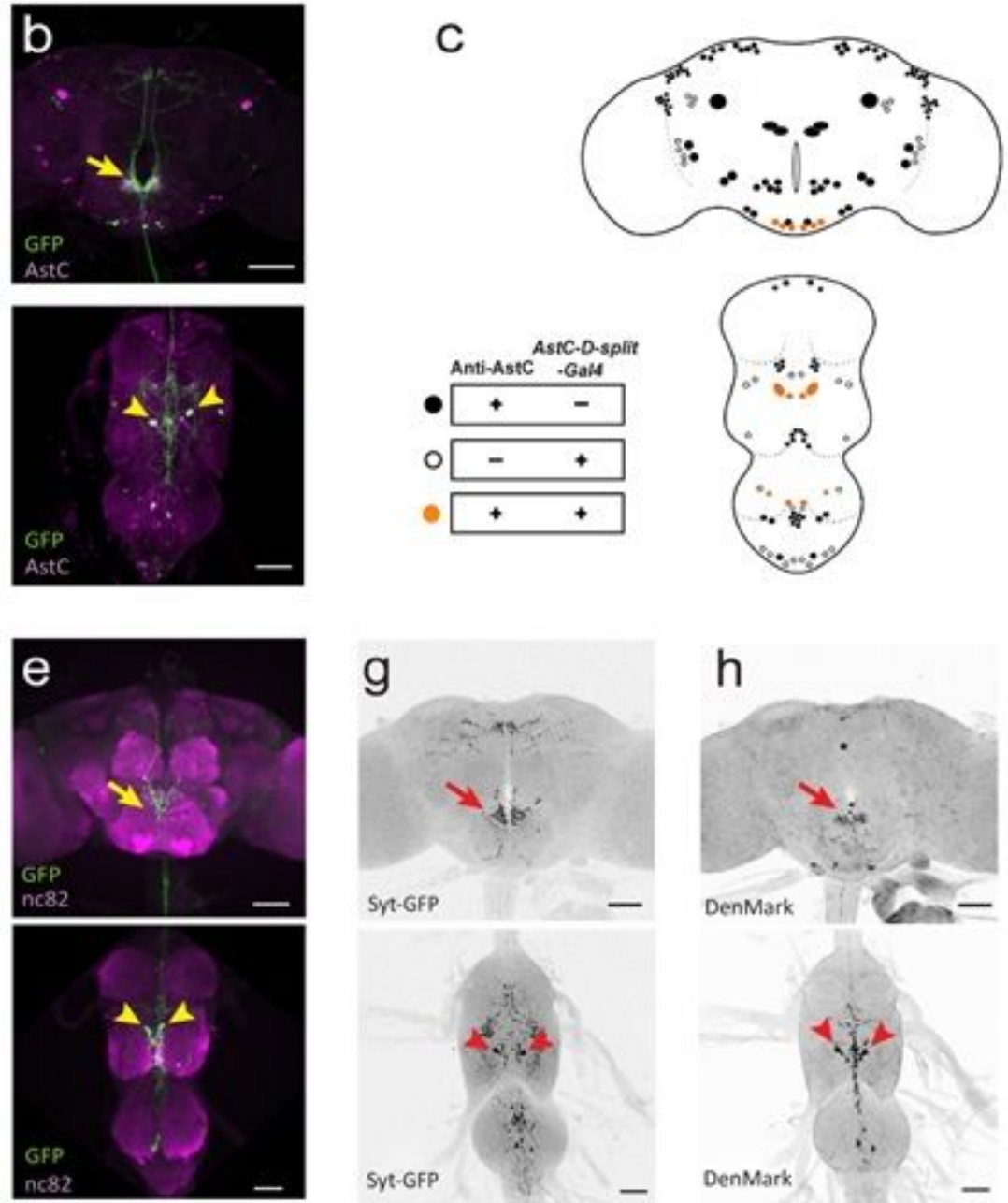

f
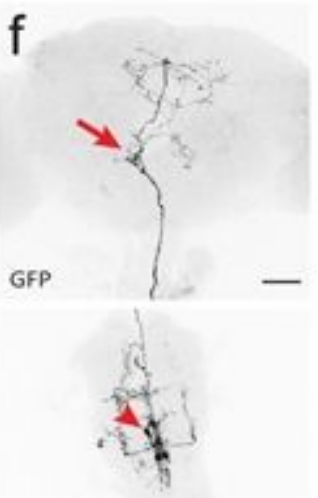

GFP

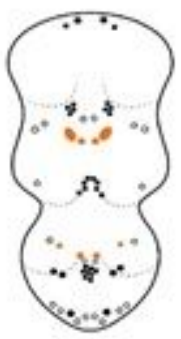

h
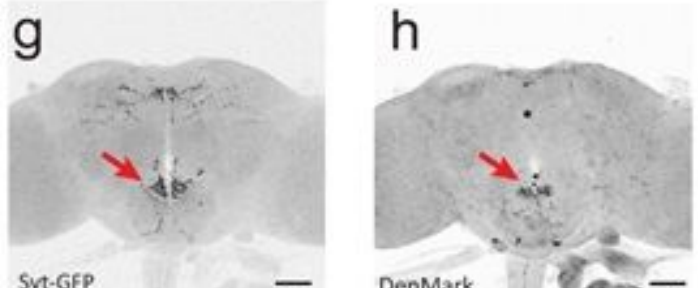

DenMark
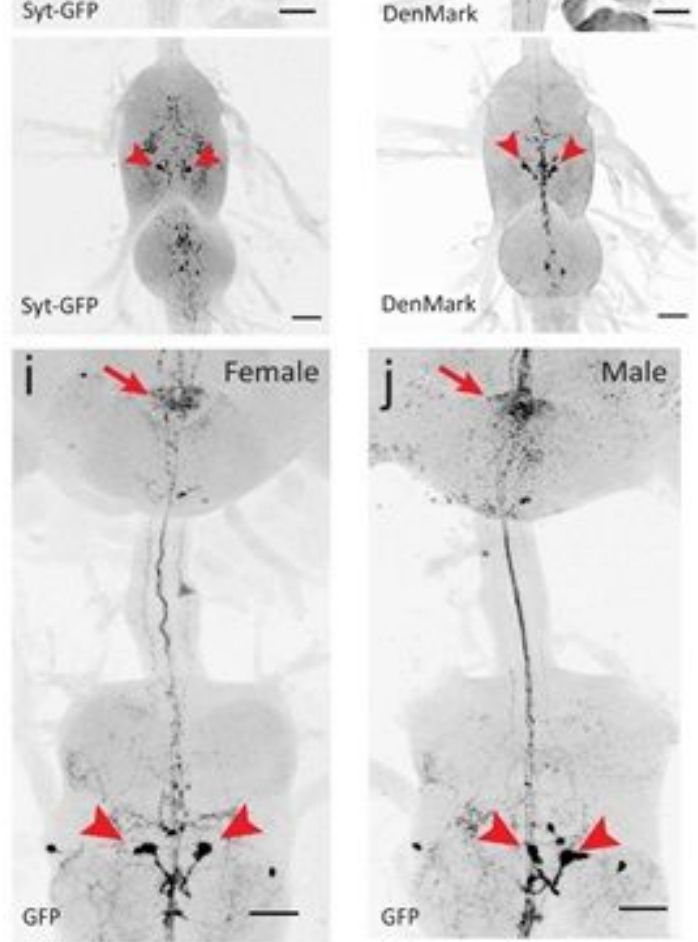

Figure 2 
Identification and characterization of AstC-mTh neurons. a, d Above, experimental protocol for a and d. Bottom, number of mature eggs per virgin female of the indicated genotypes 3 days after eclosion at $30^{\circ} \mathrm{C}(\mathrm{n}=30-40)$. One-way ANOVA followed by Tukey's test for multiple comparisons (a and $\left.d\right) ; * \star * p<$ 0.001 for comparisons against other groups (genotypes 2-5 for a and d); ns (non-significance) $p>0.05$. b Confocal Z-projection images of the brain (above) and VNC (bottom) of 4-day-old virgin females carrying AstC-D-split-Gal4 and UAS-mCD8-EGFP stained with anti-AstC (magenta) and anti-GFP (green). Arrowheads indicate two evident neurons in the VNC that project into the dorsal region of the SEZ (yellow arrow). Scale bars, $50 \mu \mathrm{m}$. c A schematic indicating anti-AstC and AstC-D-split-Gal4-positive neurons (orange circles), AstC-D-split-Gal4-positive neurons lacking anti-AstC (open circles), and anti-AstC neurons lacking AstC-D-split-Gal4 activity (closed circles). e Confocal Z-projection images of the brain (upper) and VNC (lower) of 4-day-old virgin females carrying AstC-D-split-Gal4 and TRIC transgenes (i.e., UASmCD8::RFP, LexAop2-mCD8::GFP; nSyb-MKII::nlsLexADBD;UAS-p65AD::CaM) stained with nc82 antibody (magenta) and anti-GFP (green). TRIC labels two AstC-mTh neuron somas in the VNC (arrowheads) that project into the dorsal region of the SEZ (yellow arrow). Scale bars, $50 \mu \mathrm{m}$. $f$ Negative images of TRIC labelling (anti-GFP) of single AstC-mTh neuron in the brain CNS of 4-day-old virgin females, indicating intracellular Ca2+ transients, TRIC driven by AstC-D-split-Gal4 labeled one of two AstC-mTh neurons. Arrow indicates SEZ region. Arrowheads (red) indicate AstC-mTh neuron somas. Scale bars, $50 \mu \mathrm{m} . \mathrm{g}$ Negative images of the brain (above) and VNC (bottom) of 4-day-old virgin females carrying AstC-D-splitGal4 and UAS-Syt-EGFP stained with anti-GFP. Presynaptic domains labeled by Syt-EGFP are evident in the dorsal SEZ region (arrow). Arrowheads indicate AstC-mTh neuron somas. Scale bars, $50 \mu \mathrm{m}$. $\mathrm{h}$ Negative images of the brain (above) and VNC (bottom) of 4-day-old virgin females carrying AstC-D-splitGal4 and UAS-DenMark stained with anti-RFP. Postsynaptic domains labeled by DenMark are evident in the dorsal SEZ region (arrow) and AstC-mTh neuron somas (arrowheads). Scale bars, $50 \mu \mathrm{m}$. i, j Negative images of the CNS of 4-day-old virgin female (i) and male (j) carrying AstC-D-split-Gal4 and UAS-myrEGFP stained with anti-GFP. Arrow indicates SEZ region. Arrowheads indicate AstC-mTh neuron somas. Scale bars, $50 \mu \mathrm{m}$. 

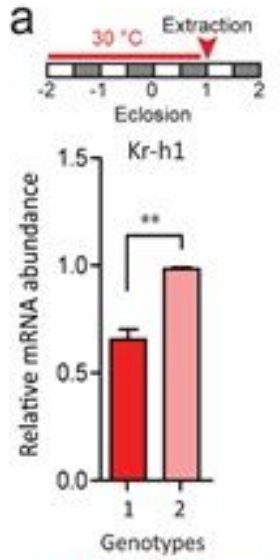

1: AstC-D-split-Gal4/UAS-dTrpA1 2: + /UAS-dTrpa1 b

Eggs in ovaries (virgin) $30^{\circ} \mathrm{C}$

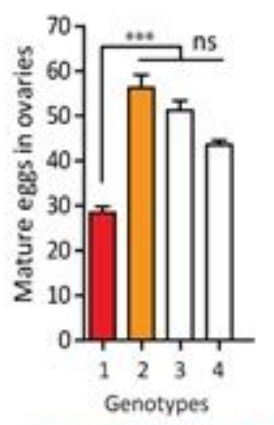

1: AstC-D.split-Gol4/UAS dTrpA1 2: AstC-D-split-Gol4/UAS-dTrpAi Methoprene

3: AstC-D-split-Gal4/t

4: +/UAS-OTrpA1

e

Follicles in ovaries (virgin) $25{ }^{\circ} \mathrm{C}$

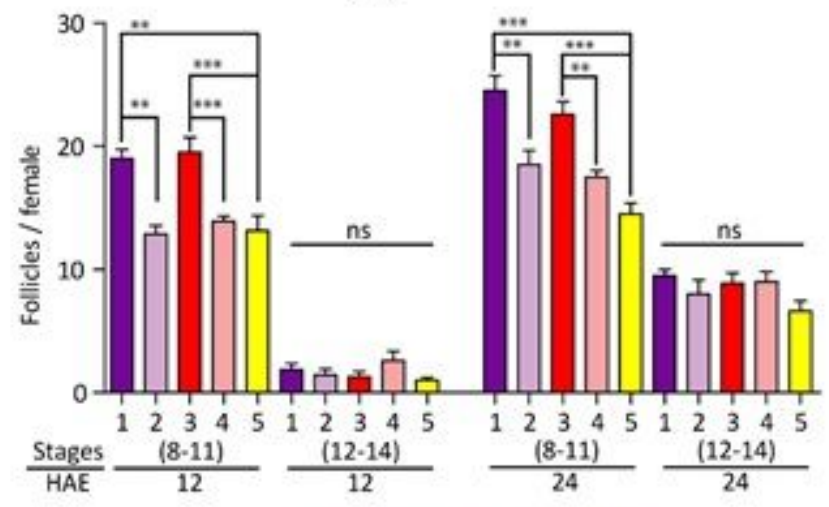

1: JHAMT-Gal4>UAS-AstCR1-IR

2: +>UAS-AstCR1-IR

Genotypes 3: JHAMT-Gal4>UAS-AstCR2-IR

4: +>UAS-AStCR2-IR

5: JHAMT-Gal4>+
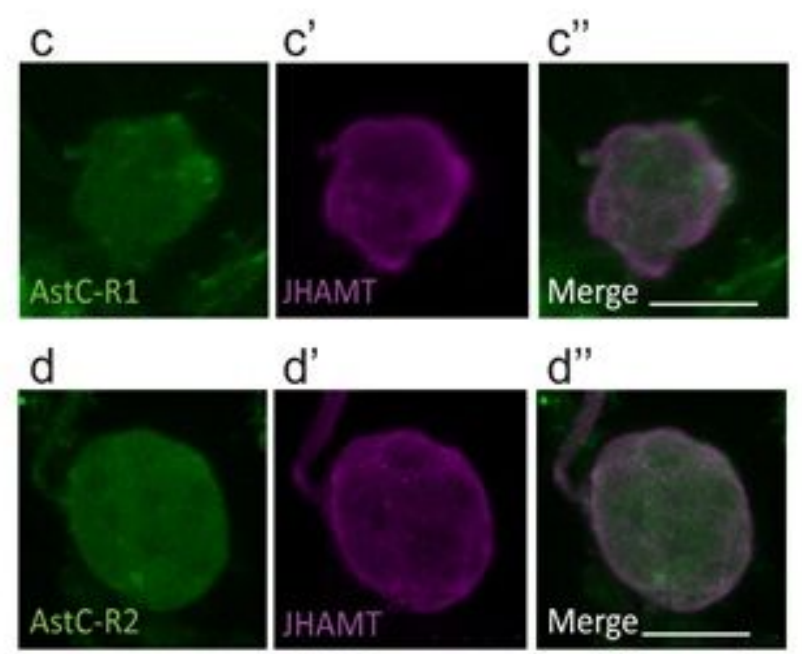

f

Eggs in ovaries (virgin) $25^{\circ} \mathrm{C}$

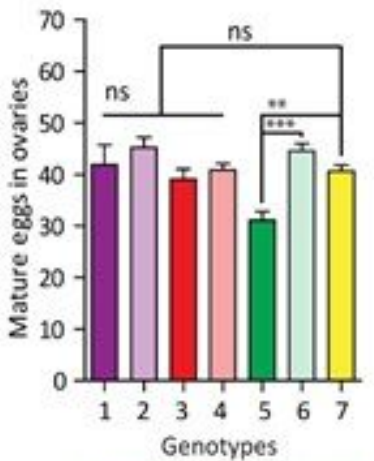

1: JHAMT-Gold > UAS-AstCR1 2: +>UAS-AStCR1

3: JHAMT-GaI4>UAS-AstCR2

4: †>UAS-AstCR2

5: JHAMT-GaI4>UAS-AStCR1-R2

6: +>UAS-AStCR1-R2

9: JHAMT-Gal4>+

\section{Figure 3}

AstC-mTh neurons regulate JH biosynthesis via AstC-R1 and AstC-R2 in the CA. a Above, experimental protocol. Below, Kr-h1 transcript levels in adult females of the indicated genotypes maintained for 3 days post-eclosion at $30^{\circ} \mathrm{C}$. Unpaired t-tests; ${ }^{\star \star} p<0.005$. b Number of mature eggs per virgin female of the indicated genotypes 3 days post-eclosion at $30^{\circ} \mathrm{C}(n=20-40)$. Genotype 2 was fed methoprene while the others were fed the vehicle control. ANOVA followed by Tukey's test for multiple comparisons (b, e, and f); $\star \star \star * p<0.001 ;{ }^{* \star} p<0.005$; ns (non-significance) $p>0.05$. c, d The CA of 4-day-old virgin females carrying AstC-R1-Gal4 (c) or AstC-R2-Gal4 (d) and UAS-mCD8-EGFP stained with anti-JHAMT (magenta) and antiGFP (green). Scale bars, $25 \mu \mathrm{m}$. e Number of stage 8-11 and 12-14 follicles per virgin female of the indicated genotypes at the indicated number of hours after eclosion (HAE) $(n=10-12)$. $f$ Number of mature eggs per virgin female of the indicated genotypes 3 days post-eclosion at $25^{\circ} \mathrm{C}(n=40-60)$. 
a

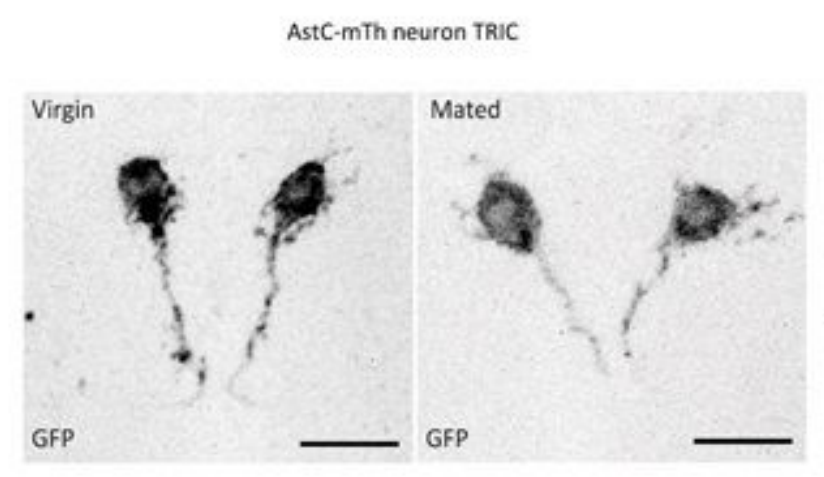

b

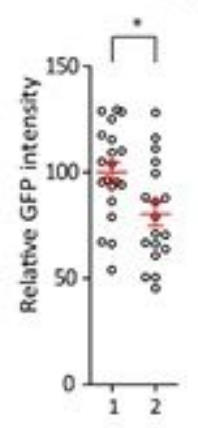

AstC-mTh neuron TRIC

1. Astc-0-solit-Golo/7h

2. AstC-D-split-Gal4/TRIC mated with CS

3. AstC-D-split-Gol4/TRIC mated with SPO/D130

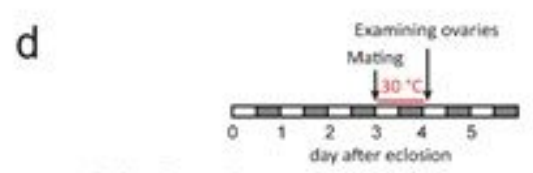

e
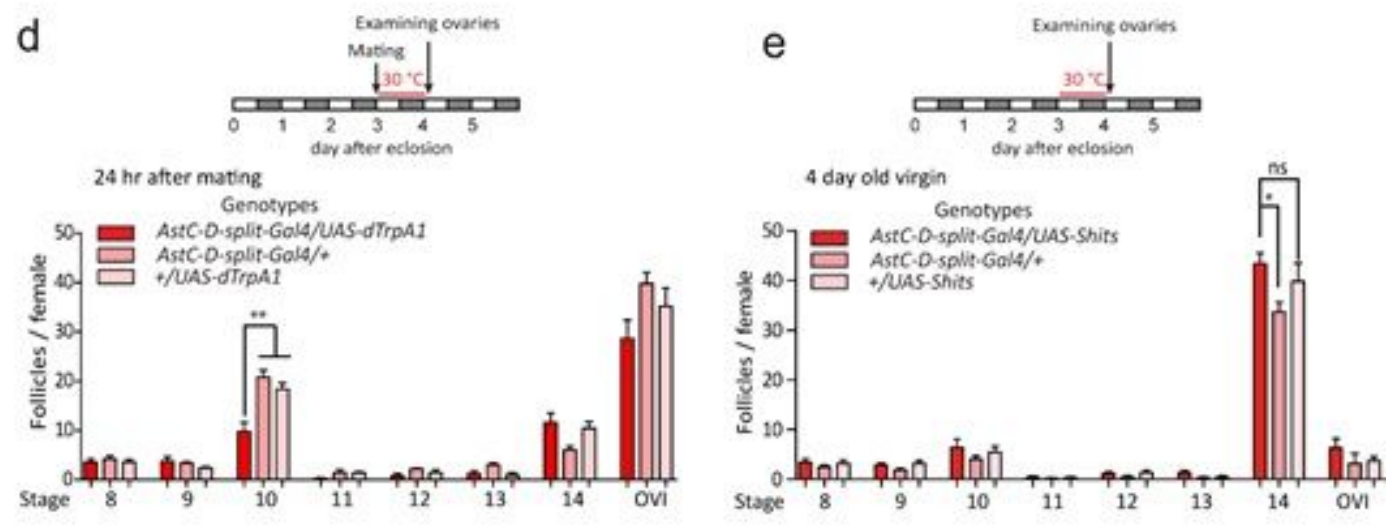

f

Examining ovaries

Matine

\section{$130^{\circ} \mathrm{C}$}
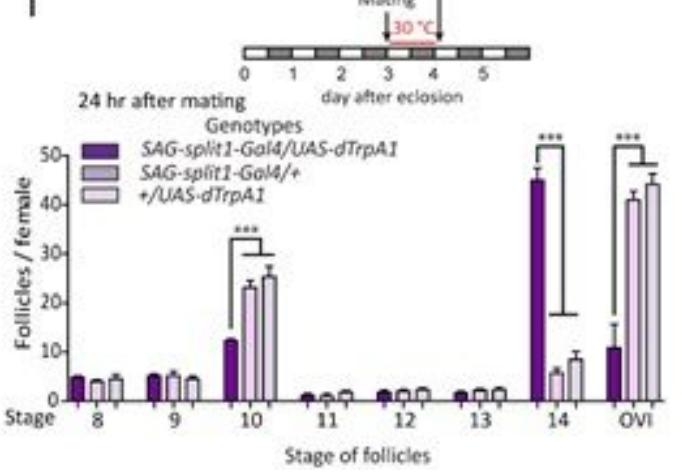

\section{Figure 4}

AstC-mTh neurons gate mating-induced vitellogenesis in response to SP. a Negative images of TRIC labelling in AstC-mTh neurons of virgin (left) and mated (right) females carrying AstC-D-split-Gal4 and TRIC transgenes, indicating intracellular Ca2+ transients. Scale bars, $10 \mu \mathrm{m}$. b, c GFP intensities from TRIC-expressing AstC-mTh neurons of virgin females and females mated with control Canton-S (CS) (b) or SP0/D130 (c) males. Unpaired t-tests; ${ }^{\star} p<0.05$. ns (non-significance) and no labeling, $p>0.05$. d, e, $f$ Above, experimental protocol. Number of follicles of the indicated stage and oviposited eggs (OVI) from females of the indicated genotypes 24 hours after mating ( $n=6-10$ for each genotype). One-way ANOVA followed by Tukey's test for multiple comparisons among genotypes; ${ }^{* \star} p<0.001$; ${ }^{* \star} p<0.005 ;{ }^{*} p<0.05$; ns (non-significance) and no labeling, $p>0.05$. 
a

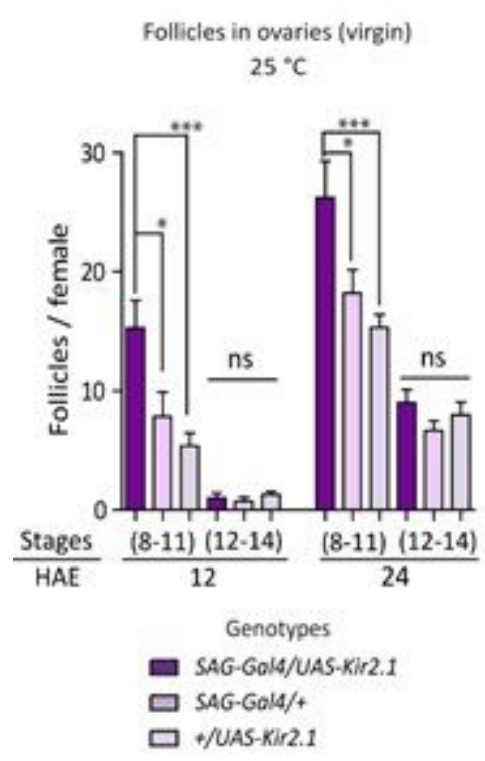

b

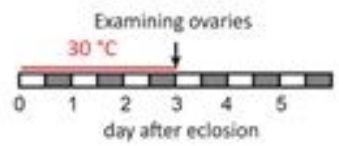

Eggs in cvaries (virgin)

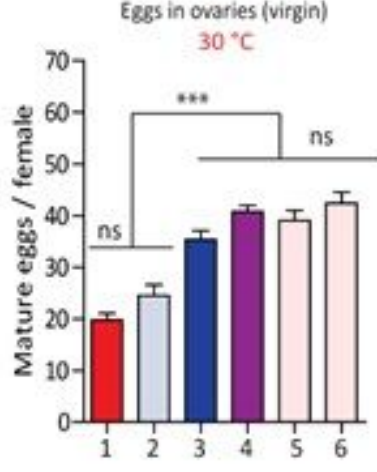

Genotypes

1: SAG Gol4|VTSO4OSVUAS-dTrpA1

2: SAG-GOISIVTSOSOSW/UAS-dTrPAI. AstC $/ 4$

3: SAG-GOL4IVTSO4OSYVAS-dTrPA1, AstC' $/$ AstC'

4: SAG-GOLGIVTSOSOSIVLAS-dTrPA1,

Methoprene

5: SAG-Gol4(VT50405)/+

6: +/UAS-dTrpA1
C

d

SAG neuron TRIC
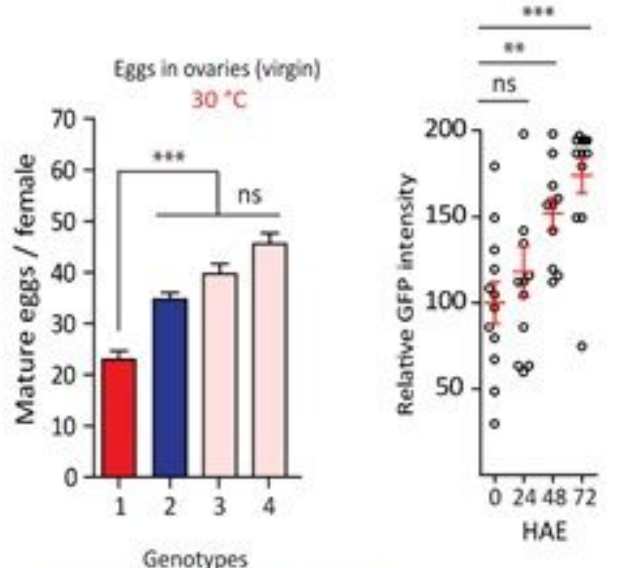

1: SAG.GaI4IVTSOAOSVIUAS-dTrpA1

2: SAG-LEXA/LEXAP-dTrPA1,

AstC-D-Gol4/UAS-Kirz.1

3: + /UAS-dTrpA1

4: + /UAS-Kir2.1

\section{Figure 5}

SAG neurons function upstream of AstC-mTh neurons and gate vitellogenesis during reproductive maturation. a Number of stage 8-11 and 12-14 follicles per virgin female of the indicated genotypes at the indicated hours after eclosion (HAE) ( $n=8-12$ for each genotype). One-way ANOVA followed by Tukey's test for multiple comparisons (a-d); ${ }^{\star \star \star} p<0.001$; ${ }^{\star \star} p<0.005 ;{ }^{*} p<0.05$; ns (non-significance) $p>$ 0.05. b, c Above, experimental protocol for $b$ and $c$. Number of mature eggs per virgin female of the indicated genotypes 3 days after eclosion under $30^{\circ} \mathrm{C}(n=40-60$ for each genotype). $d$ GFP intensities from SAG neurons of TRIC virgin females show $\mathrm{Ca} 2+$ activity at the indicated hours after eclosion (HAE).

\section{Supplementary Files}

This is a list of supplementary files associated with this preprint. Click to download.

- Supplementalinformation.docx 